\title{
MULTIAGENT DECISION MAKING IN COLLABORATIVE DECISION NETWORKS BY UTILITY CLUSTER BASED PARTIAL EVALUATION
}

\author{
YANG XIANG \\ School of Computer Science, University of Guelph, 50 Stone Road East \\ Guelph, Ontario, N1G 2W1, Canada \\ yxiang@uoguelph.ca \\ FRANK HANSHAR \\ Medicalis, 508 Riverbend Drive \\ Kitchener, Ontario, N2K 3S2, Canada \\ frank.hanshar@gridltd.ca
}

\begin{abstract}
We consider optimal multiagent cooperative decision making in stochastic environments. The focus is on simultaneous decision making, during which agents cooperate by limited communication. We model the multiagent system as a collaborative decision network $(\mathrm{CDN})$. Several techniques are developed to improve efficiency for decision making with CDNs. We present an equivalent transformation of CDN subnets to facilitate model manipulation. We propose partial evaluation to allow action profiles evaluated with reduced computation. We decompose a CDN subnet, based on clustering of utility variables. A general simultaneous decision making algorithm suite is developed that embeds these techniques. We show that the new algorithm suite improves efficiency by a combination of a linear factor and an exponential factor.
\end{abstract}

Keywords: Multiagent reasoning, simultaneous decision making, graphical models, collaborative decision networks

\section{Introduction}

A number of frameworks for cooperative multiagent decision making exist. ${ }^{1-7} \mathrm{We}$ consider multiagent, cooperative, simultaneous decision making in partially observable and stochastic environments. Here, simultaneous signifies that each agent decides on actions over multiple decision variables at once. One example problem is collaborative design in supply chains, ${ }^{8}$ where multiple manufacturers cooperate to design a product, each manufacturer is responsible for designing one component, local designs are subject to local and inter-component constraints, and are to be globally optimized. Design must take into account uncertainty on materials, manufacturing processes, product deployment conditions, etc., and optimization must consider preferences of all manufacturers, as well as those of consumers.

In general, complexity of simultaneous decision making is exponential on the total number of decision variables. A general approach for tractability is to ex- 
plore conditional independence and factorization through graphical models. ${ }^{9}$ Multiply sectioned Bayesian networks $(\mathrm{MSBNs})^{10,11}$ are among the earliest multiagent graphical models for probabilistic reasoning. A key idea pioneered in MSBNs is to organize agents into a hypertree with running intersection, according to conditional independence. The hypertree decomposition is used subsequently in other probabilistic graphical models, e.g., OOBNs, ${ }^{12}$ and some Dec-MDP frameworks. ${ }^{13}$ $\mathrm{CDNs}^{8 \mathrm{a}}$ are extensions of MSBNs, from probabilistic to decision theoretic, and also explore the hypertree. In addition to hypertree, MSBNs also pioneered inter-agent message decomposition, through linkage trees ${ }^{14}$ for a second level of factorization, resulting in further improvement of efficiency. Neither OOBNs, nor Dec-MDP frameworks ${ }^{13}$ nor existing CDN frameworks, ${ }^{15}$ explore such message decomposition. One contribution of this work is to enable exploration of inter-agent message decomposition in CDNs, through utility clusters (Section 5).

We model simultaneous decision problems through CDNs, whose main assumptions are the following. The multiagent system (whose state is described by a collection of variables) is decomposed into overlapping subsystems, that are organized into a hypertree with running intersection, according to certain conditional independence (elaborated later). Each subsystem is hosted by an agent $A_{i}$, where $i$ indexes agent, and consists of decision variables (action choices of $A_{i}$ ), chance variables (environmental conditions or consequences of actions), and utility variables (preference of $A_{i}$ ). Dependency among variables in each subsystem is modeled as a decision subnet.

In simultaneous decision making, each agent decides an action, for each local decision variable. The collection of local actions specifies a local action profile of the agent. The collection of actions over all decision variables of all agents specifies a joint action profile, which is equivalent to joining local action profiles of all agents. The objective of simultaneous decision making in CDN is to find a joint action profile, that maximizes the total expected utility for the agent team. Applying to collaborative design, a joint action profile represents a product design, consisting of a design for each component. A local action profile is the corresponding design of one component, to be supplied by one manufacturer.

A method for optimal decision making in CDNs was proposed earlier. ${ }^{15}$ Its computational complexity is linear on the number of agents, but exponential on the number of decisions per subsystem. In this work, we propose novel techniques, that improve the decision making efficiency, while maintaining optimality. Our contributions include the following. First, existing CDN specification requires agent interfaces (variables shared between subsystems) to be made of decision variables only. It is motivated by efficiency consideration, ${ }^{15}$ but it limits the expressiveness. We extend $\mathrm{CDN}$ agent interfaces to include additional chance variables. The extension widens applicability of CDNs, while maintaining the computational advantage

${ }^{\mathrm{a}} \mathrm{CDN}$ was proposed with the name collaborative design network. Due to its generality, it is renamed later, keeping the abbreviation. 
of existing agent interfaces. Secondly, we propose a compact, equivalent transformation of CDNs, where each directed path starts from a decision variable, followed by a chance variable, and ends with a utility variable. The compact transformation facilitates development and execution of novel techniques described below. Third, we propose a technique, called partial evaluation, that replaces the normal evaluation of a local action profile by a more efficient computation for non-optimal action profiles, whenever possible. It improves decision making efficiency by a linear factor. Finally, we present a mechanism for inter-agent message decomposition, called utility clusters, which further improves decision making efficiency exponentially.

The remainder of the paper is organized as follows: Section 2 introduces and extends the CDN graphical models. The compact, equivalent transformation of CDNs is developed in Section 3. Section 4 introduces partial evaluation for local decision making by individual agents. The utility cluster based partial evaluation is developed in Section 5, where local decision making is integrated into multiagent decision making. Experimental evaluation of the new algorithm suite is reported in Section 6. Section 7 relates the current work to the literature. To ease reader's burden on formal notations, they are summarized in Appendix.

\section{Collaborative Decision Networks}

This section introduces CDNs, while extending the earlier representation. ${ }^{8,15}$ Problem definition is given in Section 2.1. Decomposition of a cooperative multiagent system is considered in Section 2.2. Graphical modeling of subsystems is presented in Section 2.3. Existing decision method for (unextended) CDNs is overviewed in Section 2.4.

\subsection{Problem definition}

A cooperative multiagent system consists of an environment, populated by a set $\mathcal{A}$ of $n$ agents. The environment state is described by a collection $\mathcal{E}$ of chance variables. Each $e_{i} \in \mathcal{E}$ has a finite domain $E f_{i}=\left\{e_{i 1}, e_{i 2}, \ldots\right\}$. Denote the maximum domain cardinality by $\kappa=\max _{i}\left|E f_{i}\right|$. A proper subset of $\mathcal{E}$ may be observed by agents.

Let $\mathcal{D}$ be a collection of decision variables. Each $d_{i} \in \mathcal{D}$ has a finite domain of options or actions $O p_{i}=\left\{d_{i 1}, d_{i 2}, \ldots\right\}$. Denote the maximum domain cardinality by $\sigma=\max _{i}\left|O p_{i}\right|$. If a decision is made by a single agent, it is a private decision. Otherwise, it is a shared decision, made cooperatively by two or more agents. Each configuration of $\mathcal{D}$ describes a joint action profile of agents.

Let $\mathcal{U}$ be a collection of utility variables, such that each is associated with a single agent. Each $u_{i} \in \mathcal{U}$ is dependent on a set $\pi_{i} \subset \mathcal{E}$ of chance variables, through a utility function $u_{i}\left(\pi_{i}\right) \in[0,1]$. Denote the maximum cardinality of $\pi_{i}$ by $m=\max _{i}\left|\pi_{i}\right|$. Utilities in $\mathcal{U}$ are assumed additively independent. Each $u_{i}$ is assigned a weight $w_{i} \in(0,1)$, and weights of utility variables for the same agent sum to one. The weighted sum $\sum_{i} w_{i} u_{i}\left(\pi_{i}\right)$ over all utility variables of the same agent encodes preference of the agent over environment states. Each $A_{j} \in \mathcal{A}$ is 
associated with an agent weight $a w_{j} \in(0,1)$, such that $\sum_{j} a w_{j}=1$, which encodes relative significance of preference among agents. $\mathcal{U}$, together with agent weights and utility weights, describe preference state of the system.

The multiagent system state is described by the collection $\mathcal{V}=\mathcal{D} \cup \mathcal{E} \cup \mathcal{U}$ of variables. The system $\mathcal{V}$ is decomposed into overlapping subsystems $V_{1}, \ldots, V_{n}$, where each $V_{i}$ is hosted by agent $A_{i} \in \mathcal{A}$. Just as a decision variable may be shared or private, a chance variable may be shared or private. When a private variable is observed (chance) or instantiated (decision), the observation or instantiation is also private. On the other hand, every utility variable is private to a particular agent.

For any agent $A_{i}$ with subsystem $V_{i}=D_{i} \cup E_{i} \cup U_{i}$, a local action profile is a configuration over any subset $S D \subseteq D_{i}$ of decision variables. We refer to an action profile that is neither local nor joint as a partial action profile.

Let $\overline{j d}$ be a joint action profile, and $\bar{d}_{i}$ be the projection of $\overline{j d}$ over $D_{i}$, denoted $\bar{d}_{i}=\operatorname{proj}\left(\overline{j d}, D_{i}\right)$. In other words, $\bar{d}_{i}$ is the local action profile over $D_{i}$ that is consistent with $\overline{j d}$. The expected utility of $\overline{j d}$ is

$$
e u(\overline{j d} \mid o b s)=\sum_{i} a w_{i}\left(\sum_{j} w_{i j} P\left(\pi_{i j} \mid \bar{d}_{i}, o b s\right) u_{i j}\left(\pi_{i j}\right)\right),
$$

where $i$ indexes subsystem, $j$ indexes utility variable in $i$ th subsystem, $\pi_{i j}$ denotes the parent set of utility $u_{i j}$, and obs denotes the set of observed chance variable values by all agents. The objective of CDN decision making is to determine an optimal joint action profile

$$
\overline{j d}^{*}=\arg \max _{\overline{j d}} e u(\overline{j d} \mid o b s),
$$

which we refer to as simultaneous decision making.

Example 1. (Collaborative design on supply chain) The set $\mathcal{E}$ consists of environment variables, that describe properties of raw materials, manufacturing processes, and product deployment conditions, as well as those that describe objective performance measures (e.g., cost of a material, max speed of a car) of the product under design. The set $\mathcal{D}$ consists of design parameters (e.g., material of casing, max memory capacity of a laptop) of the product. The set $\mathcal{U}$ consists of utility variables that quantify subjective preference of stakeholders over product performance measures.

Although the joint action profile obtained by simultaneous decision making is often executed at the same time, as in Example 1, it does not have to be the case.

Example 2. (Multiagent expedition - $\mathrm{MAE}^{16}$ ) A team of mobile agents explore a unknown open area (a grid), with objects of various types. Each agent can sense a small radius, but not agents and objects beyond. It can move to a cell, and manipulate the object there. Successful manipulation may involve cooperation, which requires agents to meet at the cell. Effects of actions (movement and manipulation) are uncertain. An agent receives a reward at a cell, whose value depends on the 
object at the cell (encoded by cell type), the action performed, and degree of success in manipulation. To maximize team reward, agents need to choose behavior wisely, among weak avoidance of unproductive cells, strong avoidance of harmful cells, independent operation, or cooperation.

$\mathcal{E}$ consists of agent positions ( $p s)$, position cell types $(c t)$, and effects of manipulation $(e f)$. $\mathcal{D}$ consists of movement decisions $(m v)$ of each agent, and private object manipulation decisions $(p d)$. $\mathcal{U}$ consists of rewards $(r w)$. Each $m v$ action moves an agent from the current cell to an adjacent cell. More than one movement may be needed for nearby agents to meet and cooperate. Hence, a joint action profile needs to specify a short sequence of movements for each agents. To this end, variables may be temporally indexed. We index agents by subscripts, and temporal steps $t=0, \ldots, T$ by superscripts, where $T$ is the number of steps (a small integer, e.g., $T=2)$. For instance, after observing current positions $p s_{i}^{0}(i=1, \ldots, n)$, decision on $m v_{i}^{1}$ will be made, which leads to new positions $p s_{i}^{1}$.

We consider alternative executions of joint action profile from simultaneous decision making in MAE: (1) interleaving decision making for $T=1$ with executing the resultant joint action; (2) interleaving decision making for $T=2$ with execution; (3) interleaving decision making for $T=2$ with executing the resultant joint action for $t=1$ only.

The joint action in case (1) is optimal for $T=1$, but may not be optimal from the perspective of $T=2$. The joint action for $t=1$ in case (2) is optimal for $T=2$, but the joint action for $t=2$ may not be optimal. This is because the joint action for $t=2$ is conditioned on observation at $t=0$, but not on observation available at $t=1$. Hence, case (3) is superior than both (1) and (2). It executes the joint action for $t=1$, which is optimal from the perspective of $T=2$.

We assume that when simultaneous decision making with CDN is applied to problems that involve multiple temporal steps, it is used as case (3), and $T$ is a small integer.

\subsection{System decomposition}

We assume that system decomposition satisfies a hypertree condition, defined below. In the definition, a junction tree is a cluster tree, where the intersection of any two clusters is contained in every cluster, on the path between the two.

Definition 1. (Hypertree) Decomposition of $\mathcal{V}$ into $V_{1}, \ldots, V_{n}$ forms a hypertree, if the following holds.

(1) Intersection (if non-empty) between any $V_{j}$ and $V_{k}(i \neq k)$ consists of only (shared) decision variables and chance variables.

(2) A junction tree exists with $V_{1}, \ldots, V_{n}$ as its clusters.

We refer to any separator in the hypertree $\mathcal{H}$ as an agent interface. For disjoint sets $X, Y, Z$ of variables, denote conditional independence (CI) of $X$ and $Y$ given $Z$ 
by $I(X, Z, Y) .{ }^{9}$ System decomposition into hypertree is assumed to observe decision and observation induced CI (DOICI), defined below and illustrated in Fig. 1.

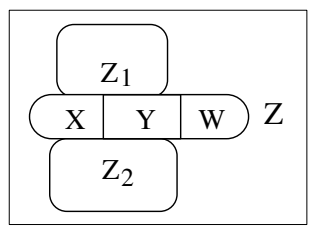

Fig. 1. Illustration of DOICI

Definition 2. (Decision and observation induced CI) Let $Z=X \cup Y \cup W$ be an agent interface, that separates hypertree $\mathcal{H}$ into subtrees $\mathcal{H}_{1}$ and $\mathcal{H}_{2}$, where $X \subset \mathcal{D}$ is a set of decision variables, $Y$ is a set of observed chance variables, and $W$ is a set of additional chance variables $(Y, W \subset \mathcal{E}$ and $Y \cap W=\emptyset)$. Let $Z_{1}$ and $Z_{2}$ be collections of decision and chance variables, excluding $Z$, located in $\mathcal{H}_{1}$ and $\mathcal{H}_{2}$, respectively. Then interface $Z$ has DOICI property, if the following holds.

(1) $Y$ is observed before decision making;

(2) $I\left(Z_{1}, Z, Z_{2}\right)$; (3) $I\left(Z_{1}, X \cup Y, W\right)$; and (4) $I\left(Z_{2}, X \cup Y, W\right)$.

In earlier works in $\mathrm{CDNs},{ }^{8,15}$ agent interfaces are assumed as being made of decisions only, namely, $X$ above. It was shown that, although interfaces made of chance variables allow distributed optimal decision making, they cannot reduce computational complexity, relative to centralized decision making. On the other hand, interfaces made of decisions not only support distributed optimal decision making, but also allows significant efficiency improvement, over centralized decision making. Defs. 1 and 2 extend the interface composition to include chance variables, $Y \cap W$, subject to DOICI, as specified in Def. 2. The extension allows chance variables in the interface, if either they are observed, i.e., $Y$, or they are independent given shared decisions $X$ and observed chances $Y$, i.e., $W$. The extension enhances expressiveness of CDNs, making them more widely applicable, while preserving the advantage analyzed earlier. ${ }^{15}$ Additional illustration of Defs. 1 and 2 can be found in Examples 2 and 3, after introducing subsystem modeling.

\subsection{Subsystem graphical models}

For each subsystem $V=D \cup E \cup U$, where $D \subset \mathcal{D}, E \subset \mathcal{E}$, and $U \subset \mathcal{U}$, variables in an agent interface are shared, and the rest are private. Denote $\rho=|D|$ and $\eta=|U|$ (these notations are summarized in Appendix). The subsystem is modeled as a decision subnet $S=(D, E, U, G, P, T)$. $G$ is a connected acyclic directed graph (DAG), whose nodes are labeled by elements of $V$. It encodes dependence and conditional independence in $V$, through four types of legal arcs: decision to decision 
(for decision constraints), decision to chance (for effects of decisions), chance to chance, and chance to utility.

Because a utility is intended to evaluate desirability of actions, every parent (a chance) of every utility must have a decision ancestor. Because an action must be judged on its expected utility, each decision must have a utility descendant. Because a chance is intended to model the uncertain consequence of actions, each chance must be on an undirected path to a decision. These topological requirements are summarized below:

Definition 3. (Subnet topology) A subnet structure $G$ satisfies the following:

(1) For every $u \in U$, each parent of $u$ must have an ancestor $d \in D$.

(2) Every $d \in D$ must have a descendant $u \in U$.

(3) For every $e \in E$, there must be an undirected path from $e$ to a $d \in D$.

Each chance node $e_{i}$ is associated with a conditional probability table (CPT) $P\left(e_{i} \mid \delta_{i}\right)$, conditioned on its parent set $\delta_{i}$. Denote the maximum size of such parent set by $m=\max _{i}\left|\delta_{i}\right|$. Each non-root decision node is associated with a CPT (made of extreme probabilities only), expressing a decision constraint (forbidding some action profiles). $P$ is the set of CPTs, one for each node in $D \cup E$. T is the set of utility functions, one for each node in $U$.

A CDN is a tuple $(\mathcal{A}, \mathcal{V}, \mathcal{H}, \mathcal{S})$, where $\mathcal{S}$ is the set of decision subnets, one for each subsystem. Example 3 below illustrates a CDN. Before the example, we note several differences between CDN subnets and well known influence diagrams (IDs).

Due to the above restriction on legal arcs, a decision node in a subnet can only have decision parents. This differs from IDs. For sequential decision making, IDs allow chance parents for decision nodes, signifying observations available prior to making each decision. CDNs are intended for simultaneous decision making, and there is no essential need for such encoding. Any prior information that constrains the domain for any $d_{i} \in \mathcal{D}$ is assumed to be reflected in $O p_{i}$ accordingly.

Furthermore, although both CDNs and IDs allow decision parents for decision nodes, their syntax and semantics are different. IDs allow a decision node to have a past decision parent. Since CDNs are for simultaneous decision making, such encoding of temporal sequence between decisions is not needed. Instead, arcs between decision nodes in CDNs express atemporal constraints. In addition, arcs between decision nodes in IDS are not associated with any numerical parameter, while in CDNs, they are associated with numerical CPTs.

Example 3. Decision making in MAE (Example 2) can be modeled with a CDN. Fig. 2 illustrates a CDN with $T=2$. Physical formation of an agent team can be maintained, using techniques known as cooperation frame and agent sphere. ${ }^{16}$ They influence agent movements, so that their interactions, e.g., meeting, are regulated. As the result, the hypertree in (c) is valid under DOICI. Details on these techniques can be found from the above reference. 


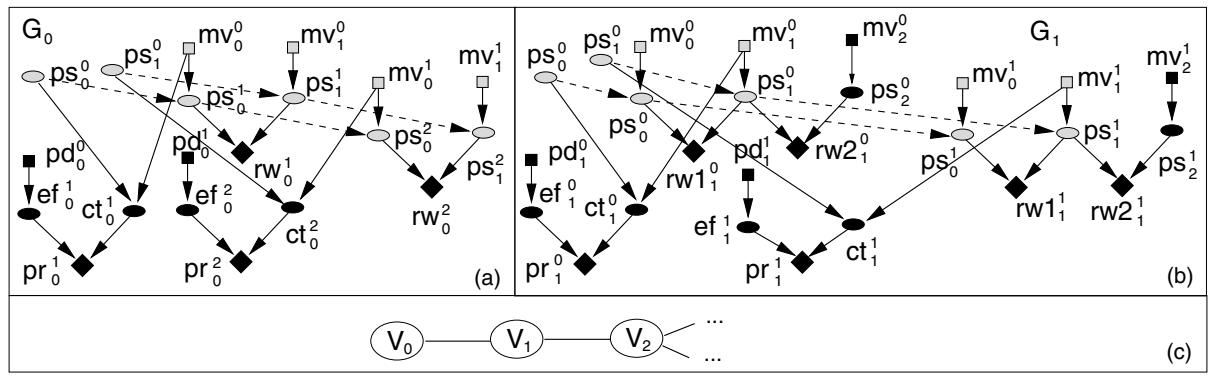

Fig. 2. (a) CDN subnet for agent $A_{0}$. (b) Subnet for $A_{1}$. (c) Hypertree.

Agent $A_{0}$ is adjacent to $A_{1}$ on hypertree, and Fig. 2 (a) shows its subnet. Nodes in $V_{0}, E_{0}, U_{0}$ are drawn as squares, ovals, diamonds, respectively. Subscripts index agents and superscripts index temporal steps. Movement decisions $(m v)$ of both $A_{0}$ and $A_{1}$ are modeled, as well as two private object manipulation decisions $(p d)$. Agent positions ( $p s)$ and position cell types $(c t)$ are dependent on movement actions. Temporal dependency is shown by dashed arcs.

Variables shared by $A_{0}$ and $A_{1}$ are shaded. The interface is decomposed into $Z=X \cup Y \cup W$ according to Def. 2 as follows:

$$
X=\left\{m v_{0}^{0}, m v_{1}^{0}, m v_{0}^{1}, m v_{1}^{1}\right\}, \quad Y=\left\{p s_{0}^{0}, p s_{1}^{0}\right\}, \quad W=\left\{p s_{0}^{1}, p s_{1}^{1}, p s_{0}^{2}, p s_{1}^{2}\right\},
$$

where $Y$ is observed prior to decision making. Note that agent interface cannot contain $Y$ and $W$, according to. ${ }^{8,15}$ However, they are necessary for $A_{0}$ and $A_{1}$ to reason about meeting and cooperation. They are enabled by Defs. 1 and 2 .

\subsection{Decision making}

A distributed optimal decision method for CDNs was developed earlier. ${ }^{15}$ First, the expected utility of each local action profile over $D_{i}$ is evaluated by the corresponding agent $A_{i}$. Then, pairwise inter-agent communication occurs in two rounds, initiated by an arbitrary agent $A_{0}$. In round one, messages flow along hypertree towards $A_{0}$, starting from leaf agents. For each sending agent $A_{i}$, the message contains a utility evaluation of each local action profile over its interface with the receiving agent $A_{j}$, based on the subtree rooted at $A_{i}$. In the second round, messages flow away from $A_{0}$. For each sending agent $A_{j}$, the message contains an optimal local action profile, over its interface with the receiving agent $A_{i}$.

After communication, an optimal joint action profile is determined. By exploring conditional independence encoded in the hypertree, the decision method reduces the time complexity from being exponential in $|\mathcal{D}| \approx n \rho$, to being linear in $n$ and exponential in $\rho .^{15}$

CDNs are extensions of MSBNs, ${ }^{14}$ a framework for multiagent probabilistic reasoning, to decision theoretic reasoning. In addition to hypertree decomposition, MSBNs also pioneered inter-agent message decomposition (using so-called linkage 
trees), for a second level of factorization, resulting in further improvement of efficiency. Decision method in earlier work of $\mathrm{CDNs}^{15}$ is unable to explore such message decomposition.

In this work, we propose a novel mechanism for message decomposition in CDNs, called utility clusters, that allows an exponential efficiency improvement. Utility clusters are closely related to linkage trees, but are significantly different. They are significantly different from the graph-theoretical perspective, but both improve efficiency by decomposing inter-agent message. Linkage trees do so by factorizing probabilistic messages multiplicatively in MSBNs, while utility clusters decompose utility messages in CDNs additively.

As introduced above, existing method for simultaneous decision making in CDNs starts by computing the expected utility of each local action profile. In this work, we propose another technique, called partial evaluation, to replace such an evaluation by a less expensive computation for non-optimal action profiles, whenever possible. Partial evaluation allows a linear efficiency improvement. We propose an algorithm suite to integrate partial evaluation and utility clustering, to enable combined efficiency gain, while maintaining optimality of simultaneous decision making.

\section{Length-2 CDN Subnets}

The new algorithm suite we propose is directly applicable to CDNs, whose subnets are in a regular form, or can be converted equivalently to such form. In Section 3.1, we restrict CDN subnets to a form where decision nodes must be roots. In Section 3.2, we further restrict subnets and define the regular form. We illustrate how a subnet that does not conform to the regular form is transformed into one in Section 3.3.

\subsection{Decision rooted subnets}

First, we regulate decision nodes in a subnet.

Definition 4. (Decision rooted subnet) A subnet is decision rooted, if all its decision nodes are root nodes.

For example, subnets in Fig. 2 are decision rooted. The only restriction of rooted subnet is that it disallows a decision node from having other decision parents, which is legal in a general CDN subnet. We show below that such syntactic restriction is not semantically limiting: An arc between a decision node and its decision parents signifies a constraint between these decisions. Decision nodes involved in such a constraint can be semantically equivalently combined.

Example 4. Let $d_{1} \in\left\{d_{10}, d_{11}\right\}$ and $d_{2} \in\left\{d_{20}, d_{21}\right\}$ be two decisions in a subnet, with an arc from $d_{2}$ to $d_{1}$. Decision $d_{2}$ can be freely made, denoted by a uniform CPT $P\left(d_{2}\right)$. The CPT $P\left(d_{1} \mid d_{2}\right)$ associated with $d_{1}$ is

$$
P\left(d_{10} \mid d_{20}\right)=0.5, \quad P\left(d_{10} \mid d_{21}\right)=1 .
$$


That is, $d_{1}$ can be freely made when $d_{2}=d_{20}$, but action $d_{11}$ is prohibited when $d_{2}=d_{21}$. The two decisions can be combined into a single decision

$$
d \in\left\{\left(d_{10}, d_{20}\right),\left(d_{11}, d_{20}\right),\left(d_{10}, d_{21}\right)\right\} .
$$

Although the above combination may lose some representational clarity, it is semantically equivalent: specifying the same set of decision options. To facilitate development and implementation of the techniques presented below, we assume that such combination has been performed recursively for each internal decision node. Hence, we consider only decision rooted CDN subnets in the remainder.

\subsection{Regular length-2 subnets}

Next we define a regular form for CDN subnets.

Definition 5. (Regular length-2 subnet) A subnet is regular length-2, if the following holds.

(1) All decision nodes are root nodes and vice versa.

(2) All utility nodes are leaf nodes and vice versa.

(3) Every directed path from a root to a leaf has length 2.

Note that the first condition is stronger than decision rooted. It follows that a regular length- 2 subnet must be decision rooted. The inverse, however, is not always true. Fig. 3 shows a regular length-2 subnet. Subnet in Fig. 2 (a) is not regular length-2, as it contains chance roots $p s_{0}^{0}$ and $p s_{1}^{0}$, and length-3 paths from $m v_{0}^{0}$ and $m v_{1}^{0}$ to $r w_{0}^{2}$. We will sometimes refer to regular length-2 subnets simply as being length- 2 .

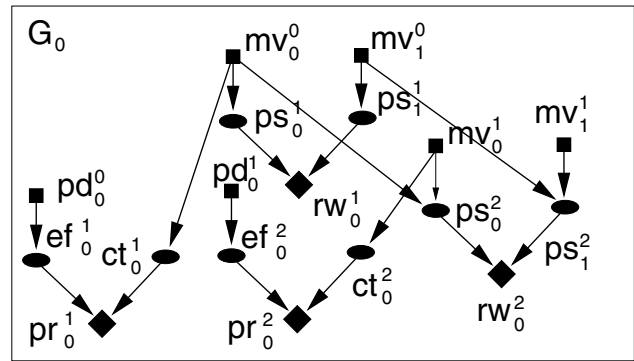

Fig. 3. A regular length-2 subnet

A subnet that is not length-2 may be transformed equivalently into length-2.

Definition 6. (Decision equivalent subnets) Two decision rooted subnets are decision equivalent, if the following holds:

(1) They have the same set of decision variables. 
(2) They have the same set of utility variables.

(3) Expected utility of each local action profile computed from one subnet is identical to that from the other.

We illustrate below how a subnet that is not regular length-2 can be decision equivalently transformed into regular length-2, using the subnet in Fig. 2 (a) as an example. A general characterization of length-2 equivalent subnets and a general transformation procedure are beyond the scope of this work.

\subsection{Equivalent subnet transformation: illustration}

First, remove the arc from observed chance node $p s_{0}^{0}$ to $c t_{0}^{1}$ equivalently. The arc from $p s_{0}^{0}$ to $p s_{0}^{1}$ can be removed similarly. Then $p s_{0}^{0}$ is isolated without impact to local action profile evaluation, and can be removed equivalently. Chance $p s_{1}^{0}$ can be removed similarly.

To illustrate removal of arc from $p s_{0}^{0}$ to $c t_{0}^{1}$, the subnet fragment, including $p s_{0}^{0}$, $c t_{0}^{1}, m v_{0}^{0}$, and $p r_{0}^{1}$, is depicted in Fig. 4 (a) with variable names simplified. Encoding

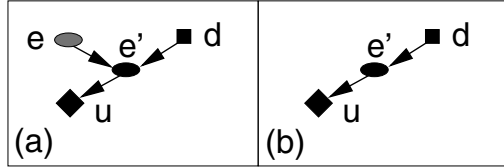

Fig. 4. (a) A subnet fragment that is not length-2. (b) Length-2 fragment after chance root removal.

observation on $e$ by $P(e \mid o b s)$, the arc from $e$ to $e^{\prime}$ can be equivalently removed, by replacing $\mathrm{CPT} P\left(e^{\prime} \mid e, d\right)$ at node $e^{\prime}$ with

$$
P\left(e^{\prime} \mid d, o b s\right)=\sum_{e} P\left(e^{\prime} \mid d, e\right) P(e \mid o b s) .
$$

Using the above technique, $p s_{0}^{0}$ and $p s_{1}^{0}$ (as well their outgoing arcs) can be removed equivalently from Fig. 2 (a). The resultant subnet is shown in Fig. 5 (a). It is still not length-2, due to length-3 paths from $m v_{0}^{0}$ and $m v_{1}^{0}$ to $r w_{0}^{2}$.

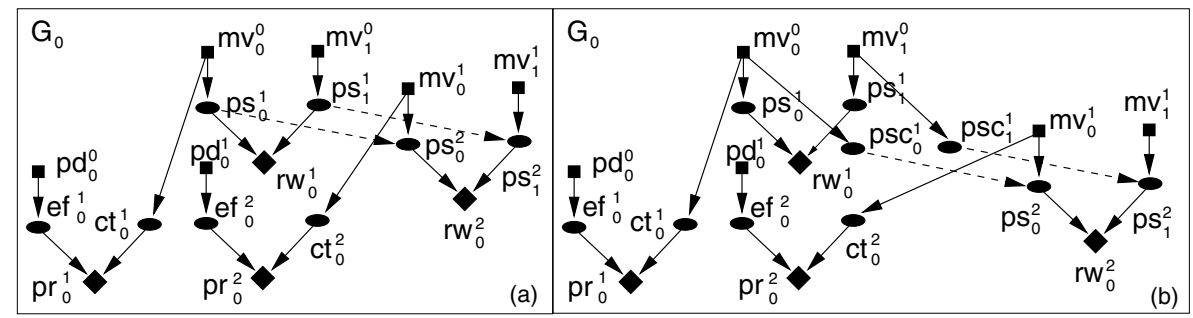

Fig. 5. (a) Subnet after removing chance roots. (b) Subnet after chance node duplication. 
To reduce the two paths to length-2, first create duplicate copies of $p s_{0}^{1}$ and $p s_{1}^{1}$, shown as $p s c_{0}^{1}$ and $p s c_{1}^{1}$ in (b). It is equivalent as the expected utility of $m v_{0}^{0}$ and $m v_{1}^{0}$ through $r w_{0}^{1}$ and $r w_{0}^{2}$ are additively independent. Next, merge the chance nodes between $m v_{0}^{0}$ and $r w_{0}^{2}$, and do the same for the other length-3 path.

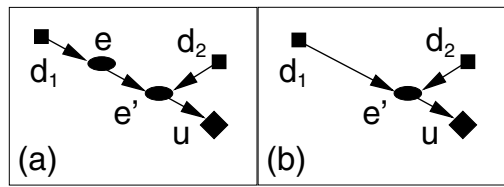

Fig. 6. (a) A subnet fragment that is not length-2. (b) Length-2 fragment after merging chance nodes.

To illustrate merging of $p s c_{0}^{1}$ and $p s_{0}^{2}$, the subnet fragment, including $m v_{0}^{0}, p s c_{0}^{1}$, $p s_{0}^{2}, m v_{0}^{1}$, and $r w_{0}^{2}$, is depicted in Fig. 6 (a) with simplified variable names. Chance $e$ can be merged into $e^{\prime}$ as shown in (b), by replacing CPT $P\left(e^{\prime} \mid e, d_{2}\right)$ at $e^{\prime}$ with

$$
P\left(e^{\prime} \mid d_{1}, d_{2}\right)=\sum_{e} P\left(e^{\prime} \mid e, d_{2}\right) P\left(e \mid d_{1}\right) .
$$

After merging chance nodes in the two length-3 paths, subnet in Fig. 5 (b) is decision equivalently transformed into the subnet in Fig. 3. In the remainder, we assume that subnets of CDNs are either regular length-2, or have been converted decision equivalently to length-2. We focus on developing a more efficient method for simultaneous decision making in such CDNs. Since every chance variable in a length-2 CDN has a non-empty subset of decision variables as its parents, we refer to chance variables in length-2 CDNs as effect variables.

\section{Partial Evaluation in Local Decision Making}

This section presents partial evaluation for more efficient action profile evaluation in regular length-2 subnets. A local action profile is normally evaluated by computing its expected utility. The basic idea of partial evaluation is to replace the evaluation by a less expensive computation for non-optimal action profiles, whenever possible. Section 4.1 introduces partial evaluation through a trivial decision subnet, with a single decision and a single utility. The method is generalized to subnets with multiple decisions in Section 4.3, and to subnets with multiple utilities in Section 4.4. The algorithms presented form the basis for local computation at individual agents, during simultaneous decision making.

\subsection{Single decision variable}

First, we introduce partial evaluation through a trivial decision subnet, where $\rho=1$, $\eta=1$, and $V=\left\{d_{i}, e_{i}, u_{i}\right\}$. That is, the DAG is $d_{i} \rightarrow e_{i} \rightarrow u_{i}$. The expected utility 
of taking action $d_{i}=d_{i j}$ is

$$
e u\left(d_{i j}\right)=P\left(e_{i 1} \mid d_{i j}\right) u_{i}\left(e_{i 1}\right)+P\left(e_{i 2} \mid d_{i j}\right) u_{i}\left(e_{i 2}\right)+\ldots,
$$

which requires $\kappa$ probability retrievals, $\kappa$ utility retrievals, $\kappa$ multiplications, and $\kappa-$ 1 additions. Action $d_{i j}$ is fully evaluated, when $e u\left(d_{i j}\right)$ is computed. Its complexity is $O(\kappa)$. To compute the optimal action $\left(d_{i k}^{*}\right.$, meuv), where

$$
d_{i k}^{*}=\arg \max _{j} e u\left(d_{i j}\right) \quad \text { and } \quad \text { meuv }=e u\left(d_{i k}^{*}\right),
$$

it generally involves full evaluation of each $d_{i j}$, with $O(\sigma \kappa)$ complexity.

When there is no confusion, we refer to an effect value $e_{i j} \in E f_{i}$ simply as an effect. For every action, we refer to an effect value with the highest probability (indexed once and referenced repeatedly) as the pivot effect of the action.

Definition 7. (Pivot effect) Let $d_{i k}$ be an action of $d_{i}$ in the subnet $d_{i} \rightarrow e_{i} \rightarrow u_{i}$. Let $e_{i k}$ be an effect value such that

$$
P\left(e_{i k} \mid d_{i k}\right)=\max _{j} P\left(e_{i j} \mid d_{i k}\right) .
$$

Then $e_{i k}$ is the pivot effect of $d_{i k}$. If the condition holds for multiple effect values, one is selected arbitrarily.

Note that effect $e_{i k}$ is obtained by varying $j$ in $e_{i j}$, until $P\left(e_{i j} \mid d_{i k}\right)$ is maximal. We denote the pivot effect of action $d_{i k}$ by $e_{i k}$, and refer to $P\left(e_{i k} \mid d_{i k}\right)$ as the pivot probability of $d_{i k}$. Consider MAE, for example, where decision move has alternative actions $m_{-} n$ (move north), $m_{-} s, m_{-} e, m_{-} w$, and halt. Its effect landing has alternative values $l_{-} n$ (land north), $l_{-} s, l_{-} e, l_{-} w$, and $s_{-} p$ (same place). Table 1 shows the CPT $P$ (landing $\mid$ move $)$. From the third row, the pivot effect of $m \_n$ is $l \_n$, and the pivot probability of $m \_n$ is 0.90 .

Table 1. Illustration of pivot assumption with CPT P(landing $\mid$ move)

\begin{tabular}{lllll|l}
\hline \hline \multicolumn{5}{c|}{ landing } & \\
\hline$l \_n$ & $l_{-} s$ & $l_{\_} e$ & $l_{\_} w$ & $s_{-} p$ & move \\
\hline 0.90 & 0.03 & 0.03 & 0.03 & 0.01 & $m_{-} n$ \\
0.03 & 0.90 & 0.03 & 0.03 & 0.01 & $m_{-} s$ \\
0.03 & 0.03 & 0.90 & 0.03 & 0.01 & $m_{\_} e$ \\
0.03 & 0.03 & 0.03 & 0.90 & 0.01 & $m_{-} w$ \\
0.025 & 0.025 & 0.025 & 0.025 & 0.90 & halt \\
\hline \hline
\end{tabular}

If we denote the maximum utility of $u_{i}$ as $u_{i}^{\max }=\max _{x} u_{i}\left(e_{i x}\right)$, it follows that

$$
e u\left(d_{i k}\right) \leq P\left(e_{i k} \mid d_{i k}\right) u_{i}\left(e_{i k}\right)+\left(1-P\left(e_{i k} \mid d_{i k}\right)\right) u_{i}^{\max } \equiv Q_{i k} .
$$


Note that utility function $u_{i}($.$) normally spans the range [0,1]$ fully, in which case $u_{i}^{\max }=1$. Our method does not depend on this condition, and hence we keep $u_{i}^{\max }$ explicit.

Action $d_{i j}$ is said to dominate $d_{i k}$, iff $e u\left(d_{i j}\right)>e u\left(d_{i k}\right)$. If $Q_{i k}<e u\left(d_{i j}\right)$, then $e u\left(d_{i k}\right)<e u\left(d_{i j}\right)$ and $d_{i k}$ is dominated by $d_{i j}$. Computing $Q_{i k}$ requires one pivot probability retrieval, two utility retrievals, two multiplications, and two additions. Action $d_{i k}$ is partially evaluated, when $Q_{i k}$ is computed.

Suppose pivot probabilities of alternative actions of $d_{i}$ are nearly identical, e.g., Table 1, where $P\left(l_{-} n \mid m_{-} n\right)=P\left(l_{-} e \mid m_{-} e\right)=P\left(s_{-} p \mid\right.$ halt $)=0.90$. As another example, a message may be sent by email or post (alternative actions). Emails most likely arrive in seconds (pivot effect of action emailing), while post mails most likely arrive in days (pivot effect of post_mailing). P(arrive_in_seconds|emailing) and $P$ (arrive_in_days|post_mailing) (pivot probabilities) are similar.

Formally, we make the following pivot assumption:

$$
\forall j, k P\left(e_{i j} \mid d_{i j}\right)=P\left(e_{i k} \mid d_{i k}\right) \equiv p, \quad(j \neq k) .
$$

That is, for any effect, its pivot probabilities for distinct actions are identical.

Partial evaluation of action $d_{i k}$ amounts to computing

$$
Q_{i k}=p u_{i}\left(e_{i k}\right)+(1-p) u_{i}^{\max } .
$$

To determine dominance of $d_{i j}$ over $d_{i k}$ and, in particular, whether

$$
Q_{i k}=p u_{i}\left(e_{i k}\right)+(1-p) u_{i}^{\max }<e u\left(d_{i j}\right)
$$

holds, we check equivalently whether

$$
u_{i}\left(e_{i k}\right)<\frac{e u\left(d_{i j}\right)}{p}-\frac{1-p}{p} u_{i}^{\max }
$$

holds. We assume that $d_{i j}$ has been fully evaluated, and the right hand side (threshold) has been obtained before $d_{i k}$ is evaluated. If the above inequality holds, $d_{i k}$ is dominated by $d_{i j}$, it can be rejected with just one utility retrieval and one comparison, and the threshold can be reused for evaluating the next action. Hence, partial evaluation of $d_{i k}$ has $O(1)$ complexity.

This leads to partial evaluation based decision for computing $\left(d_{i k}^{*}\right.$, meuv) as follows:

(1) Apply full evaluation to the first action and establish threshold.

(2) For each alternative action, apply partial evaluation, and reject it if so warranted.

(3) Otherwise, apply full evaluation to it and update threshold.

(4) Select the last accepted action as $d_{i k}^{*}$.

Using this procedure, efficiency is gained by evaluating a $d_{i k}$ fully, only if the above inequality fails. Let $\theta \in(0,1)$ be the percentage of $d_{i k}$ fully evaluated. Then the complexity of partial evaluation based decision is $O(\theta \sigma \kappa+\sigma)$. 


\subsection{Examples for single decision variable}

We illustrate partial evaluation based decision with examples.

Example 5. Consider a trivial decision subnet with DAG $d_{i} \rightarrow e_{i} \rightarrow u_{i}, d_{i} \in$ $\{0,1,2,3,4\}, e_{i} \in\{0,1,2,3,4\}$, and parameters in Table 2 . The optimal action

Table 2. CPT $P\left(e_{i} \mid d_{i}\right)$ (left) and utility function $u_{i}\left(e_{i}\right)$ (right) for Example 5

\begin{tabular}{lllll|l}
\hline \hline \multicolumn{5}{c|}{$e_{i}$} & \\
\hline 0 & 1 & 2 & 3 & 4 & $d_{i}$ \\
\hline 0.09 & 0.07 & 0.01 & 0.80 & 0.03 & 0 \\
0.09 & 0.04 & 0.80 & 0.05 & 0.02 & 1 \\
0.80 & 0.02 & 0.03 & 0.08 & 0.07 & 2 \\
0.06 & 0.04 & 0.03 & 0.80 & 0.07 & 3 \\
0.80 & 0.04 & 0.02 & 0.01 & 0.13 & 4 \\
\hline \hline
\end{tabular}

\begin{tabular}{l|l}
\hline \hline$d_{i}$ & $u_{i}\left(e_{i}\right)$ \\
\hline 0 & 0.6 \\
1 & 0.2 \\
2 & 1.0 \\
3 & 0.0 \\
4 & 0.9 \\
\hline \hline
\end{tabular}

$\left(d_{i k}^{*}\right.$, meuv $)$ from a full evaluation is $d_{i k}^{*}=1$ and meuv $=0.88$.

The pivot assumption (Eq. 1) holds for $P\left(e_{i} \mid d_{i}\right)$ and the pivot probability is $p=0.8$. Applying partial evaluation, $d_{i 0}$ is fully evaluated to get $e u\left(d_{i}=0\right)=0.105$. For $d_{i 1}$, apply Eq. (2) to obtain estimate $Q_{i 1}=0.8 * 1.0+0.2 * 1.0=1.0>0.105$. Hence, $d_{i 1}$ is fully evaluated to get $e u\left(d_{i}=1\right)=0.88$. For $d_{i 2}$, the estimate is $Q_{i 2}=0.68<0.88$ and $d_{i 2}$ is rejected. Subsequently, $d_{i}=3$ and $d_{i}=4$ are all rejected. Hence, the resultant optimal action is $d_{i k}^{*}=1$ and meuv $=0.88$, and is identical to the result of full evaluation. Out of the five alternative actions of $d_{i}$, only two of them $\left(d_{i}=0,1\right)$ are fully evaluated.

In practice, pivot assumption may not always hold. The example below shows that the soundness of partial evaluation is not sensitive to violation of the pivot assumption.

Example 6. Consider another subnet with parameters in Table. 3. The optimal action from a full evaluation is $d_{i k}^{*}=1$ and meuv $=0.888$.

Pivot assumption does not hold in $P\left(e_{i} \mid d_{i}\right)$, as pivot probabilities for distinct $d_{i}$ values are $0.85,0.82,0.6,0.89,0.9$, respectively. Suppose the first pivot probability $p=0.85$ is used for partial evaluation. First, $d_{i 0}$ is fully evaluated to get $e u\left(d_{i}=\right.$ $0)=0.081$. For $d_{i 1}$, estimate is $Q_{i 1}=0.85 * 1.0+0.15 * 1.0=1.0>0.081$. Hence, $d_{i 1}$ is fully evaluated to get $e u\left(d_{i}=1\right)=0.888$. Subsequently, $d_{i}=2,3,4$ are all rejected. Hence, the same optimal action as full evaluation is obtained. This shows that violation of the pivot assumption does not necessarily prevent identification of the optimal action.

Although partial evaluation is not sensitive to violation of pivot assumtion, there is no guarantee that the result is truly optimal when pivot assumption does not hold, 
Table 3. CPT $P\left(e_{i} \mid d_{i}\right)$ (left) and utility function $u_{i}\left(e_{i}\right)$ (right) for Example 6

\begin{tabular}{lllll|l}
\hline \hline \multicolumn{5}{c|}{$e_{i}$} & \\
\hline 0 & 1 & 2 & 3 & 4 & $d_{i}$ \\
\hline 0.09 & 0.04 & 0.01 & 0.85 & 0.01 & 0 \\
0.07 & 0.04 & 0.82 & 0.05 & 0.02 & 1 \\
0.60 & 0.12 & 0.13 & 0.08 & 0.07 & 2 \\
0.03 & 0.01 & 0.03 & 0.89 & 0.04 & 3 \\
0.90 & 0.04 & 0.02 & 0.01 & 0.03 & 4 \\
\hline \hline
\end{tabular}

\begin{tabular}{l|l}
\hline \hline$d_{i}$ & $u_{i}\left(e_{i}\right)$ \\
\hline 0 & 0.6 \\
1 & 0.2 \\
2 & 1.0 \\
3 & 0.0 \\
4 & 0.9 \\
\hline \hline
\end{tabular}

as shown below.

Example 7. Consider another subnet with parameters in Table. 4. The optimal action from a full evaluation is $d_{i k}^{*}=1$ and meuv $=0.601$.

Table 4. CPT $P\left(e_{i} \mid d_{i}\right)$ (left) and utility function $u_{i}\left(e_{i}\right)$ (right) for Example 7

\begin{tabular}{lllll|l}
\hline \hline \multicolumn{5}{c|}{$e_{i}$} & \\
\hline 0 & 1 & 2 & 3 & 4 & $d_{i}$ \\
\hline 0.07 & 0.04 & 0.82 & 0.05 & 0.02 & 0 \\
0.30 & 0.22 & 0.13 & 0.08 & 0.27 & 1 \\
0.09 & 0.04 & 0.01 & 0.85 & 0.01 & 2 \\
0.03 & 0.01 & 0.03 & 0.89 & 0.04 & 3 \\
0.90 & 0.04 & 0.02 & 0.01 & 0.03 & 4 \\
\hline \hline
\end{tabular}

\begin{tabular}{l|l}
\hline \hline$d_{i}$ & $u_{i}\left(e_{i}\right)$ \\
\hline 0 & 0.2 \\
1 & 1.0 \\
2 & 0.6 \\
3 & 0.0 \\
4 & 0.9 \\
\hline \hline
\end{tabular}

Pivot assumption does not hold in $P\left(e_{i} \mid d_{i}\right)$. Suppose the first pivot probability $p=0.82$ is used for partial evaluation. First, $d_{i 0}$ is fully evaluated to get $e u\left(d_{i}=\right.$ $0)=0.564$. For $d_{i 1}$, estimate $Q_{i 1}=0.82 * 0.2+0.18 * 1.0=0.344<0.564$ and $d_{i 1}$ is rejected. Similarly, the subsequent 3 actions are also rejected. The final decision is $d_{i k}^{*}=0$ and meuv $=0.564$, which is suboptimal. Note that meuv $=0.564$ is fairly close to 0.601 from full evaluation.

To summarize, when pivot assumption holds, partial evaluation is guaranteed to be optimal while allowing computational savings. When the assumption does not hold, partial evaluation can still be optimal, although not guaranteed. In the experimental study (Section 6), we evaluate how likely optimal decisions can still be obtained when pivot assumption does not hold and how close the suboptimal decisions are relative to the optimal. 


\subsection{Multiple decision variables}

Next, we generalize partial evaluation to the case where $\rho>1$ and $\eta=1$. That is, multiple decision variables collectively influence a utility $u_{i}$, e.g., subnet in Fig. 7 . We first generalize pivot effect of action in Def. 7 to compound pivot effect of a local action profile.

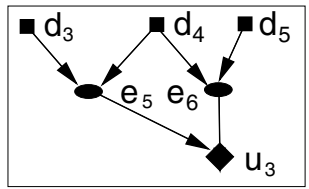

Fig. 7. A length-2 subnet where $\rho>1$ and $\eta=1$

Definition 8. (Compound pivot effect) Let $S=(D, E, U, G, P, T)$ be a regular length-2 subnet, where $\rho>1$ and $\eta=1$. Let $\bar{d}$ be a local action profile over $D$.

(1) A compound effect $\overline{e^{\prime \prime}}$ of $\bar{d}$ is a configuration over $E$.

(2) For each $e_{i} \in E$, parent set $\delta_{i} \subset D$ of $e_{i}$, and local action profile $\operatorname{proj}\left(\bar{d}, \delta_{i}\right)$, the effect value $e_{i j}$ is the pivot effect of local plan $\operatorname{proj}\left(\bar{d}, \delta_{i}\right)$ if

$$
e_{i j}=\arg \max _{k} P\left(e_{i k} \mid \operatorname{proj}\left(\bar{d}, \delta_{i}\right)\right) \text {, }
$$

breaking ties arbitrarily.

(3) The compound pivot effect $\bar{e}$ of $\bar{d}$ is the configuration of all pivot effects over E.

Example 8. For the subnet in Fig. $7, \bar{d}=\left(d_{31}, d_{42}, d_{51}\right)$ is a local action profile, and so is $\operatorname{proj}\left(\bar{d}, \delta_{6}\right)=\left(d_{42}, d_{51}\right)$. Configuration $\left(e_{51}, e_{62}\right)$ is a compound effect.

If $e_{51}$ is the pivot effect of local action profile $\left(d_{31}, d_{42}\right)$, and $e_{62}$ is the pivot effect of $\left(d_{42}, d_{51}\right)$, then $\left(e_{51}, e_{62}\right)$ is the compound pivot effect of $\bar{d}$.

Without confusion, we simply omit the word 'compound' in 'compound effect' and 'compound pivot effect'. Let $\bar{d}$ be a local action profile over $D, \bar{e}$ be its pivot effect, $\bar{e}^{\prime \prime}$ be any other effect, and $e u(\bar{d})$ be the expected utility of $\bar{d}$. Then

$$
e u(\bar{d})=P(\bar{e} \mid \bar{d}) u_{i}(\bar{e})+\sum_{\bar{e}^{\prime \prime}} P\left(\bar{e}^{\prime \prime} \mid \bar{d}\right) u_{i}\left(\bar{e}^{\prime \prime}\right) .
$$

Below, we consider a decision subtask, which will be the basis for local computation by individual agents, during simultaneous decision making. Let $\beta$ be a subset of $D$ and $\gamma=D \backslash \beta$. Let $\bar{b}$ be an action profile over $\beta, \bar{y}$ be an action profile over $\gamma$, and $(\bar{b}, \bar{y})$ be a join of action profiles. The decision subtask is to obtain a pair of functions $(\operatorname{meu}(\beta)$, peer $(\beta))$, where meu $: \beta \rightarrow[0,1]$ and peer $: \beta \rightarrow \gamma$, such that for each $\bar{b}$,

$$
\operatorname{meu}(\bar{b})=\max _{\bar{y}} e u(\bar{b}, \bar{y}) \quad \text { and } \quad \operatorname{peer}(\bar{b})=\arg \max _{\bar{y}} e u(\bar{b}, \bar{y}) .
$$


Note that $\operatorname{peer}(\bar{b})$ returns an optimal local action profile over $\gamma$, when $\beta$ is constrained to local action profile $\bar{b}$. Hence, we refer to $\beta$ as the constraint scope, and $\gamma$ as the optimization scope. In other words, the function pair $(\operatorname{meu}(\beta), \operatorname{peer}(\beta))$ specifies, for each constraint $\bar{b}$ over $\beta$, the maximum expected utility (MEU) meu $(\bar{b})$, and the corresponding optimal action profile $(\bar{b}$, peer $(\bar{b}))$ over $D$.

Example 9. Let $\beta=\left\{d_{3}, d_{4}\right\}$ and $\gamma=\left\{d_{5}\right\}$ for the subnet in Fig. 7, assuming binary decision variables. A function pair $(\operatorname{meu}(\beta), \operatorname{peer}(\beta))$ takes the following form.

\begin{tabular}{|c|cc|}
\hline $\bar{b}$ & meu $(\bar{b})$ & peer $(\bar{b})$ \\
\hline$\left(d_{31}, d_{41}\right)$ & 0.70 & $\left(d_{52}\right)$ \\
$\left(d_{31}, d_{42}\right)$ & 0.65 & $\left(d_{51}\right)$ \\
$\left(d_{32}, d_{41}\right)$ & 0.82 & $\left(d_{51}\right)$ \\
$\left(d_{32}, d_{42}\right)$ & 0.73 & $\left(d_{52}\right)$ \\
\hline
\end{tabular}

For a given local action profile $\bar{d}$ over $D$, denote $\bar{b}=\operatorname{proj}(\bar{d}, \beta), \bar{y}=\operatorname{proj}(\bar{d}, \gamma)$. Then, $\bar{d}=(\bar{b}, \bar{y})$. The decision subtask requires evaluating $e u(\bar{d})=e u(\bar{b}, \bar{y})$ for each $\bar{d}$. To evaluate $e u(\bar{d})$ for a given $\bar{d}$ by Eq. $(3), P\left(\bar{e}^{\prime \prime} \mid \bar{d}\right)$ must be computed for each $\bar{e}^{\prime \prime}$ over $E$.

Example 10. For subnet in Fig. 7, we have $P\left(\bar{e}^{\prime \prime} \mid \bar{d}\right)=P\left(e_{5}, e_{6} \mid d_{3}, d_{4}, d_{5}\right)=$ $P\left(e_{5} \mid d_{3}, d_{4}\right) P\left(e_{6} \mid d_{4}, d_{5}\right)$.

Computing $P\left(\bar{e}^{\prime \prime} \mid \bar{d}\right)$ for a particular $\bar{e}^{\prime \prime}$ involves $m$ probability retrievals and $m-1$ multiplications. There are $\kappa^{m}$ alternative $\bar{e}^{\prime \prime}$. Hence, a full evaluation of $\bar{d}$ by Eq. (3) takes $m \kappa^{m}$ probability retrievals, $\kappa^{m}$ utility retrievals, $m \kappa^{m}$ multiplications, and $\kappa^{m}-1$ additions. The complexity of a full evaluation of $\bar{d}$ is thus $O\left(m \kappa^{m}\right)$. To obtain meu $(\beta)$ by full evaluation, a total of $\sigma^{\rho}$ alternative $\bar{d}$ must be evaluated, and the complexity is thus $O\left(\sigma^{\rho} m \kappa^{m}\right)$.

For more efficient computation, we explore partial evaluation introduced in Section 4.1. First, we extend pivot assumption on a single effect variable with a single decision parent, to multiple effect variables each with multiple decision parents.

Definition 9. (Pivot assumption) Let $e_{i}$ be an effect variable, in a regular length-2 subnet $S$, with parent set $\delta_{i}$. Let $\overline{\delta_{i k}}$ be $k$ th configuration of $\delta_{i}$, and $e_{i k}$ be its pivot effect. Subnet $S$ satisfies pivot assumption if, for every $e_{i}$, the following holds:

$$
\forall j, k \quad P\left(e_{i j} \mid \overline{\delta_{i j}}\right)=P\left(e_{i k} \mid \overline{\delta_{i k}}\right), \quad(j \neq k) .
$$

The practical interpretation is that, for each effect variable, its pivot probabilities for distinct action profiles are approximately identical.

Example 11. In MAE, the pivot effect of moving north, followed by moving east, is landing on the north east location. The pivot effect of moving south, followed by moving west, is landing on the south west location. The two corresponding pivot probabilities are approximately identical. 
As shown later in our experiments, even though our decision method is derived from the pivot assumption, the method works well, when Eq. (4) holds only approximately. We observe

$$
\begin{aligned}
e u(\bar{d})= & P(\bar{e} \mid \bar{d}) u_{i}(\bar{e})+\sum_{\bar{e}^{\prime \prime}} P\left(\bar{e}^{\prime \prime} \mid \bar{d}\right) u_{i}\left(\bar{e}^{\prime \prime}\right) \leq P(\bar{e} \mid \bar{d}) u_{i}(\bar{e})+(1-P(\bar{e} \mid \bar{d})) u_{i}^{\max }, \\
& \text { where } u_{i}^{\max }=\max _{\bar{e}^{\prime \prime}} u_{i}\left(\bar{e}^{\prime \prime}\right) .
\end{aligned}
$$

It then follows from the pivot assumption that

$$
\forall \bar{d}, \bar{d}^{\prime} \quad P(\bar{e} \mid \bar{d})=P\left(\bar{e}^{\prime} \mid \bar{d}^{\prime}\right) \equiv p,
$$

where $\bar{d} \neq \bar{d}^{\prime}$, and $\bar{e}\left(\bar{e}^{\prime}\right)$ is the pivot effect of local action profile $\bar{d}\left(\bar{d}^{\prime}\right)$.

Example 12. For the subnet in Fig. 7, suppose pivot probability of $e_{5}$ given action profile $\left(d_{3}, d_{4}\right)$ is 0.7 , and that of $e_{6}$ given $\left(d_{4}, d_{5}\right)$ is 0.9. Then, from Example 10, it follows $p=0.7 * 0.9=0.63$.

When pivot assumption holds, inequation (5) becomes

$$
e u(\bar{d}) \leq p u_{i}(\bar{e})+(1-p) u_{i}^{\max } .
$$

Let $e u\left(\bar{d}^{\prime}\right)$ be given for $\bar{d}^{\prime}$. For an alternative action profile $\bar{d}$, if

$$
p u_{i}(\bar{e})+(1-p) u_{i}^{\max }<e u\left(\bar{d}^{\prime}\right),
$$

it follows that $e u(\bar{d})<e u\left(\bar{d}^{\prime}\right)$, and $\bar{d}$ is dominated by $\bar{d}^{\prime}$. Partial evaluation of $\bar{d}$ by Eq. (6) takes only two utility retrievals, with complexity $O(1)$.

Extending partial evaluation based decision in Section 4.1 with the above operation, the decision subtask to obtain $(\operatorname{meu}(\beta)$, peer $(\beta))$ can be solved by PeDecSu. In the algorithm, the scope of each for or if statement is indicated by indentation.

Algorithm 1. PeDecSu

Input: subnet over $D \cup E \cup U$ where $U=\left\{u_{i}\right\}$, max utility $u_{i}^{\max }$, pivot probability

$p$, constraint scope $\beta \subset D$, and optimization scope $\gamma=D \backslash \beta$;

Output: function pair $(\operatorname{meu}(\beta), \operatorname{peer}(\beta))$;

for each constraint $\bar{b}$ over $\beta$,

pick an action profile $\bar{y}^{\prime}$ over $\gamma$ to fully evaluate by Eq. (3) and get $e u\left(\bar{b}, \bar{y}^{\prime}\right)$;

set $m e u(\bar{b})=e u\left(\bar{b}, \bar{y}^{\prime}\right)$ and $\operatorname{peer}(\bar{b})=\bar{y}^{\prime}$;

set threshold $t h=\left(e u\left(\bar{b}, \bar{y}^{\prime}\right)-(1-p) u_{i}^{\max }\right) / p$;

for each action profile $\bar{y}$ over $\gamma$ where $\bar{y} \neq \bar{y}^{\prime}$,

retrieve $u_{i}(\bar{e})$ for pivot effect $\bar{e}$ of $\bar{d}=(\bar{b}, \bar{y})$;

if $u_{i}(\bar{e}) \geq t h$,

fully evaluate $\bar{d}$ by Eq. (3) to get $e u(\bar{d})$;

$\operatorname{meu}(\bar{b})=e u(\bar{d}), \operatorname{peer}(\bar{b})=\bar{y}$, th $=\left(e u(\bar{d})-(1-p) u_{i}^{\max }\right) / p ;$

return $(\operatorname{meu}(\beta), \operatorname{peer}(\beta))$; 
Note that when $\beta=\emptyset$, the optimization scope $\gamma=D$. In the return value of $\mathrm{PeDecSu}, \operatorname{meu}(\beta)$ becomes a single value, and $\operatorname{peer}(\beta)$ becomes a single optimal local action profile $\bar{d}^{*}$ over $D$.

Let $\theta \in(0,1)$ be the percentage of local action profiles fully evaluated by PeDecSu. Its complexity is then $O\left(\theta \sigma^{\rho} m \kappa^{m}+\sigma^{\rho}\right) \approx O\left(\theta \sigma^{\rho} m \kappa^{m}\right)$. Proposition 1 summarizes key properties of $\mathrm{PeDecSu}$, whose proof is straightforward, given the above analysis.

Proposition 1. PeDecSu satisfies the following.

(1) For each constraint $\bar{b}$ over $\beta$, meu $(\bar{b})$ is the $M E U$.

(2) For each $\bar{b}$, peer $(\bar{b})$ is the optimal action profile over $\gamma$.

(3) Its complexity is $O\left(\theta \sigma^{\rho} m \kappa^{m}\right)$.

\subsection{Multiple utility variables}

Next, we generalize partial evaluation to decision subnets where $\rho>1$ and $\eta>1$.

Example 13. Fig. 8 shows a subnet with $\eta=2$ and a weight $w_{i}$ is associated with

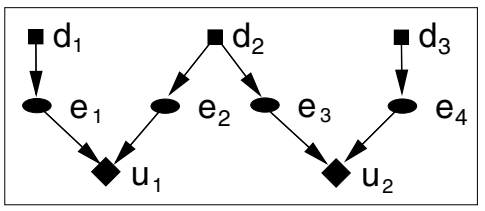

Fig. 8. A length-2 subnet where $\rho>1$ and $\eta>1$

each $u_{i}(i=1,2)$. The optimal local action profile cannot be obtained by solving the decision problem as two independent sub-problems, one over $\left\{d_{1}, d_{2}\right\}$ and the other over $\left\{d_{2}, d_{3}\right\}$. This is because the optimal action profile over $\left\{d_{1}, d_{2}, d_{3}\right\}$ may be incompatible with the optimal action profile over either $\left\{d_{1}, d_{2}\right\}$ or $\left\{d_{2}, d_{3}\right\}$. Hence, fully evaluating a local action profile over $D$ amounts to compute

$$
\begin{aligned}
& e u\left(d_{1}, d_{2}, d_{3}\right) \\
= & w_{1} \sum_{e_{1}, e_{2}} P\left(e_{1} \mid d_{1}\right) P\left(e_{2} \mid d_{2}\right) u_{1}\left(e_{1}, e_{2}\right)+w_{2} \sum_{e_{3}, e_{4}} P\left(e_{3} \mid d_{2}\right) P\left(e_{4} \mid d_{3}\right) u_{2}\left(e_{3}, e_{4}\right) .
\end{aligned}
$$

In general, let $\bar{d}$ be a local action profile over $D, \bar{e}$ be its pivot effect, $\bar{e}^{\prime \prime}$ be any alternative effect, and $e u(\bar{d})$ be expected utility of $\bar{d}$. For each utility $u_{i}(i=1, \ldots, \eta)$ with parents $\pi_{i}$, let $\alpha_{i}$ be the set of decision ancestors of $u_{i}$ (see Fig. 9). In Fig. 8, $\alpha_{1}$ for $u_{1}$ is $\left\{d_{1}, d_{2}\right\}$. Define $\overline{e_{i}}=\operatorname{proj}\left(\bar{e}, \pi_{i}\right)$, and $\overline{d_{i}}=\operatorname{proj}\left(\bar{d}, \alpha_{i}\right)$. Then, a full evaluation of $\bar{d}$ computes

$$
e u(\bar{d})=\sum_{i=1}^{\eta} w_{i}\left[P\left(\overline{e_{i}} \mid \overline{d_{i}}\right) u_{i}\left(\overline{e_{i}}\right)+\sum_{\bar{e}_{i}^{\prime \prime}} P\left(\bar{e}_{i}^{\prime \prime} \mid \overline{d_{i}}\right) u_{i}\left(\bar{e}_{i}^{\prime \prime}\right)\right] .
$$




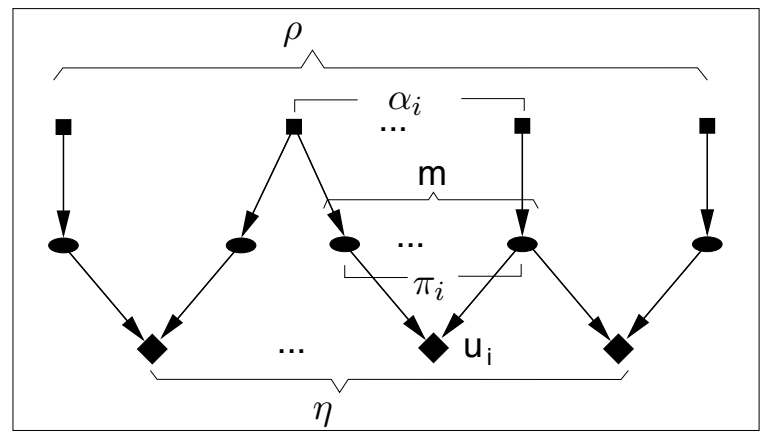

Fig. 9. Illustration of symbols

Below, we extend solution to the decision subtask in Section 4.3 to subnets where $\eta>1$. As will be seen, simultaneous decision making involves such subtasks by individual agents. Let $\beta$ be the given constraint scope, and $\gamma=D \backslash \beta$ be the optimization scope. The decision subtask is to obtain $(\operatorname{meu}(\beta), \operatorname{peer}(\beta))$ such that, for each action profile $\bar{b}$ over $\beta$,

$$
\operatorname{meu}(\bar{b})=\max _{\bar{y}} e u(\bar{b}, \bar{y}) \quad \text { and } \operatorname{peer}(\bar{b})=\arg \max _{\bar{y}} e u(\bar{b}, \bar{y}),
$$

where $\bar{y}$ is any action profile over $\gamma$.

Applying an analysis to Eq. (7) similarly as in Section 4.3, the complexity to fully evaluate $\bar{d}$ is $O\left(\eta m \kappa^{m}\right)$. The complexity to obtain meu( $\left.\beta\right)$ by full evaluation is $O\left(\sigma^{\rho} \eta m \kappa^{m}\right)$. We consider efficiency improvement with partial evaluation.

Example 14. Consider the subnet in Example 13. If $e_{j}$ is the child node of $d_{i}$, we denote its pivot effect corresponding to $d_{i k}$ by $e_{j k}$. We have

$$
\begin{aligned}
& e u\left(d_{1 x}, d_{2 y}, d_{3 z}\right) \leq \\
& w_{1} P\left(e_{1 x} \mid d_{1 x}\right) P\left(e_{2 y} \mid d_{2 y}\right) u_{1}\left(e_{1 x}, e_{2 y}\right)+w_{2} P\left(e_{3 y} \mid d_{2 y}\right) P\left(e_{4 z} \mid d_{3 z}\right) u_{2}\left(e_{3 y}, e_{4 z}\right) \\
+ & w_{1}\left(1-P\left(e_{1 x} \mid d_{1 x}\right) P\left(e_{2 y} \mid d_{2 y}\right)\right) u_{1}^{\max }+w_{2}\left(1-P\left(e_{3 y} \mid d_{2 y}\right) P\left(e_{4 z} \mid d_{3 z}\right)\right) u_{2}^{\max } .
\end{aligned}
$$

If the pivot assumption (Def. 9) holds, denote the pivot probability of $e_{i}$ by $p_{i}$. We have

$$
\begin{aligned}
e u\left(d_{1 x}, d_{2 y}, d_{3 z}\right) & \leq w_{1} p_{1} p_{2} u_{1}\left(e_{1 x}, e_{2 y}\right)+w_{2} p_{3} p_{4} u_{2}\left(e_{3 y}, e_{4 z}\right) \\
& +w_{1}\left(1-p_{1} p_{2}\right) u_{1}^{\max }+w_{2}\left(1-p_{3} p_{4}\right) u_{2}^{\max } \equiv Q .
\end{aligned}
$$

Suppose, for action profile $\left(d_{1 x}^{\prime}, d_{2 y}^{\prime}, d_{3 z}^{\prime}\right), e u\left(d_{1 x}^{\prime}, d_{2 y}^{\prime}, d_{3 z}^{\prime}\right)$ has been obtained. For action profile $\left(d_{1 x}, d_{2 y}, d_{3 z}\right)$, we compute the above value $Q$. If

$$
Q<e u\left(d_{1 x}^{\prime}, d_{2 y}^{\prime}, d_{3 z}^{\prime}\right),
$$

it follows that $e u\left(d_{1 x}, d_{2 y}, d_{3 z}\right)<e u\left(d_{1 x}^{\prime}, d_{2 y}^{\prime}, d_{3 z}^{\prime}\right)$, and $\left(d_{1 x}, d_{2 y}, d_{3 z}\right)$ is dominated by $\left(d_{1 x}^{\prime}, d_{2 y}^{\prime}, d_{3 z}^{\prime}\right)$. Partial evaluation of $\left(d_{1 x}, d_{2 y}, d_{3 z}\right)$ by Eq. (8) takes only four utility retrievals. 
In general, each utility $u_{i}$ has a set $\pi_{i}$ of effect parents (see Fig. 9). Let the effect parents be indexed by $j$. Assuming pivot assumption, each effect parent of $u_{i}$ is associated with a pivot probability $p_{i j}$. Given $e u\left(\overline{d^{\prime}}\right)$ from full evaluation of action profile $\overline{d^{\prime}}$, and an alternative action profile $\bar{d}$, if

$$
\sum_{i=1}^{\eta} w_{i}\left(\left(\prod_{j} p_{i j}\right) u_{i}\left(\overline{e_{i}}\right)+\left(1-\left(\prod_{j} p_{i j}\right)\right) u_{i}^{\max }\right)<e u\left(\overline{d^{\prime}}\right),
$$

then $\bar{d}$ is dominated by $\overline{d^{\prime}}$. This leads to threshold assignment and dominance test below:

$$
\begin{gathered}
t h=e u\left(\overline{d^{\prime}}\right)-\sum_{i=1}^{\eta} w_{i}\left(1-\left(\prod_{j} p_{i j}\right)\right) u_{i}^{\max }, \\
\sum_{i=1}^{\eta} w_{i}\left(\prod_{j} p_{i j}\right) u_{i}\left(\overline{e_{i}}\right)<t h .
\end{gathered}
$$

By pre-computing $w_{i}\left(\prod_{j} p_{i j}\right)$ for each $u_{i}$, partial evaluation of $\bar{d}$ using Eq. (10) has a complexity of $O(\eta)$. Extending PeDecSu with the above operations, PeDecMu makes partial evaluation based decision, with multiple decision and utility variables.

Algorithm 2. PeDecMu

Input: subnet over $D \cup E \cup U$, $\max$ utility $u_{i}^{\max }$ for each $u_{i}$, a pivot probability

$p_{i j}$ for each $u_{i}$ and its $j$ th parent, $\beta \subset D$ and $\gamma=D \backslash \beta$;

Output: function pair $(\operatorname{meu}(\beta), \operatorname{peer}(\beta))$;

for each constraint $\bar{b}$ over $\beta$,

pick an action profile $\overline{y^{\prime}}$ over $\gamma$ and denote $\overline{d^{\prime}}=\left(\bar{b}, \overline{y^{\prime}}\right)$;

fully evaluate $\overline{d^{\prime}}$ by Eq. (7) to get $e u\left(\overline{d^{\prime}}\right)$;

set meu $(\bar{b})=e u\left(\overline{d^{\prime}}\right), \operatorname{peer}(\bar{b})=\overline{y^{\prime}}$, and threshold th by Eq. (9);

for each action profile $\bar{y}$ over $\gamma$ where $\bar{y} \neq \bar{y}^{\prime}$,

denote $\bar{d}=(\bar{b}, \bar{y})$

test dominance by Eq. (10);

if test fails,

fully evaluate $\bar{d}$ by Eq. (7) to get $e u(\bar{d})$;

$\operatorname{meu}(\bar{b})=\operatorname{eu}(\bar{d}), \operatorname{peer}(\bar{b})=\bar{y}$, and update th by Eq. $(9) ;$

return $(\operatorname{meu}(\beta), \operatorname{peer}(\beta))$;

When $\beta=\emptyset$, the return value of $\mathrm{PeDecMu}, \operatorname{meu}(\beta)$, becomes a single value, and $\operatorname{peer}(\beta)$ becomes a single optimal action profile $\bar{d}^{*}$ over $D$. Proposition 2 summarizes key properties of PeDecMu.

Proposition 2. PeDecMu satisfies the following.

(1) For each constraint $\bar{b}$ over $\beta$, meu $(\bar{b})$ is the MEU.

(2) For each $\bar{b}$, peer $(\bar{b})$ is the optimal action profile over $\gamma$. 
(3) Its complexity is $O\left(\theta \sigma^{\rho} \eta m \kappa^{m}\right)$.

Comparing the complexity $O\left(\sigma^{\rho} \eta m \kappa^{m}\right)$ by full evaluation, PeDecMu reduces the complexity by a factor of $\theta$. If $\theta=20 \%$, then PeDecMu will be 5 times faster.

\section{Utility Cluster Based Partial Evaluation in Simultaneous Decision Making}

In this section, we present a novel technique, utility clusters, to decompose interagent messages, in the context of simultaneous decision making in regular length-2 CDNs. It is integrated seamlessly with partial evaluation. The technique decomposes messages additively, and improves efficiency exponentially from the existing method. ${ }^{15}$

Simultaneous decision making is carried out by two rounds of message passing, interleaved with local decision making by partial evaluation. The hypertree is viewed as directed from an arbitrary agent (as root), so that adjacent agents are referred to as parent and child according to the direction. In the first round, utility messages flow from leaf agents towards the root. Section 5.1 introduces utility clusters in the context of message computation by leaf agents. Message computation by general agents are considered in Section 5.2, where the clustering is extended to an arbitrary agent. In the second round, presented in Section 5.3, local action profiles over agent interfaces flow from the root agent towards leaf agents.

\subsection{Utility clusters and messages from leaf agents}

Let a leaf agent $A$ be associated with the subnet over $D \cup E \cup U$. Let the set of decision variables in its interface with the adjacent agent $B$ on hypertree be $S D \subset D$. Message $u t m_{0}(S D)$ that $A$ sends to $B$ is a MEU function that, for each local action profile $\overline{s d}$ over $S D$, specifies

$$
u t m_{0}(\overline{s d})=\max _{\overline{r d}} e u(\overline{s d}, \overline{r d}),
$$

where $\overline{r d}$ is a local action profile over $R D=D \backslash S D$. In other words, we have

$$
u m_{0}(S D)=\operatorname{meu}(S D) .
$$

From Proposition 2, it follows that, if $A$ applies PeDecMu with $\beta=S D$, the return value meu $(\beta)$ satisfies meu $(\beta)=u t m_{0}(S D)$. Since complexity of PeDecMu is exponential on $\rho$, we seek to improve its efficiency by message decomposition below.

Generally speaking, additive contributions of utility variables to $u t m_{0}(S D)$ may enable decomposition in evaluating $u t m_{0}(S D)$. On the other hand, as demonstrated at the start of Section 4.4, decomposition at the level of individual utility variables is not always feasible. We explore the existence of private decisions, that is, variables in $R D$. In the following, we classify utility variables in relation to $S D$ and $R D$, which provides a basis for sound decomposition. 
For each utility $u_{i}$ and its decision ancestors $\alpha_{i}$, we define

$$
\beta_{i}=\alpha_{i} \cap S D \quad \text { and } \quad \gamma_{i}=\alpha_{i} \backslash \beta_{i}=\alpha_{i} \cap R D .
$$

That is, $\beta_{i}$ is decision ancestors of $u_{i}$ shared between $A$ and $B$, and $\gamma_{i}$ is decision ancestors of $u_{i}$ private to $A$. Each utility variable can be classified, based on the relation between $\alpha_{i}, S D$, and $R D$, into one of four cases:

Definition 10. Let $S D$ be the set of decision variables in an agent interface, and $\alpha_{i}$ be the decision ancestor set of a utility variable $u_{i}$. Let $\beta_{i}=\alpha_{i} \cap S D$ and $\gamma_{i}=\alpha_{i} \backslash \beta_{i}$. Then $u_{i}$ is in case $k$, if the $k$ th condition below holds.

(1) $\gamma_{i}=\emptyset$.

(2) $\gamma_{i} \neq \emptyset, \beta_{i} \neq \emptyset$, and $\gamma_{i} \cap \gamma_{j}=\emptyset$ for all $u_{j}$ where $j \neq i$.

(3) $\gamma_{i} \neq \emptyset, \beta_{i} \neq \emptyset$, and $\gamma_{i} \cap \gamma_{j} \neq \emptyset$ for some $u_{j}$ where $j \neq i$.

(4) $\beta_{i}=\emptyset$.

The four cases are interpreted as follows.

Case 1: $\alpha_{i}=\beta_{i}$ is entirely shared.

Case 2: There exists no other utility variable $u_{j}$, such that $u_{i}$ and $u_{j}$ have a common private decision ancestor.

Case 3: There exists at least one utility variable $u_{j}$, such that $u_{i}$ and $u_{j}$ have a common private decision ancestor.

Case 4: $\alpha_{i}=\gamma_{i}$ is entirely private.

Example 15. Consider Fig. 10, where $S D=\left\{d_{2}, d_{3}\right\}$. Then $u_{2}$ is case $1, u_{1}$ is case $2, u_{3}$ is case 3 , and $u_{4}$ is case 4 .

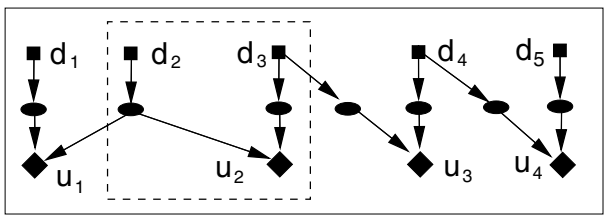

Fig. 10. Illustration of classification of utility variables

Proposition 3. The four cases of utility variable in Def. 10 are mutually exclusive and exhaustive.

Proof. The mutual exclusion is obvious. We show that they are exhaustive.

First, we have either $\beta_{i}=\emptyset$ (case 4 ) or $\beta_{i} \neq \emptyset$. Assuming $\beta_{i} \neq \emptyset$, we then have either $\gamma_{i}=\emptyset$ (case 1$)$ or $\gamma_{i} \neq \emptyset$. Next, we assume $\beta_{i} \neq \emptyset$ and $\gamma_{i} \neq \emptyset$. Then either another utility variable $u_{j}$ exists with a common private decision ancestor (case 3 ), or no such $u_{j}$ exists (case 2). 
From Proposition 3, it suffices to analyze message meu(SD) decomposition, enabled by $u_{i}$ of each case. Denote

$$
\overline{b_{i}}=\operatorname{proj}\left(\overline{s d}, \beta_{i}\right) \quad \text { and } \quad \overline{y_{i}}=\operatorname{proj}\left(\overline{s d}, \gamma_{i}\right) .
$$

[Case 1] For this case, $\overline{b_{i}}=\operatorname{proj}\left(\overline{s d}, \alpha_{i}\right)$. We refer to the subnet segment that contains $u_{i}$, all its ancestors (including $\pi_{i}$ and $\alpha_{i}$ ), and arcs among them, as the subnet segment of $u_{i}$. In Fig. 10, subnet segment of $u_{2}$ is highlighted by the dashed box.

We observe that (a) contribution of $u_{i}$ to meu(SD) can be evaluated using its subnet segment, independently of other $u_{j} \in U$, and (b) the contribution is additive. To see (a), note that contribution of $u_{i}$ to $\operatorname{meu}(S D)$ is expected utility function $e u\left(\alpha_{i}\right)$, whose evaluation is completely determined by the subnet segment of $u_{i}$. To see (b), note that if meu( $\overline{s d})=v$ is obtained using $A^{\prime}$ 's subnet, meu $(\overline{s d})=v^{\prime}$ is obtained using $A$ 's subnet with node $u_{i}$ removed, and $e u\left(\overline{b_{i}}\right)=v^{\prime \prime}$ is obtained using the subnet segment of $u_{i}$, then

$$
v=v^{\prime}+v^{\prime \prime} .
$$

Therefore, we can compute $e u\left(\alpha_{i}\right)$ using the subnet segment of $u_{i}$ according to Eq. (3). As it involves full evaluation for each $\overline{b_{i}}$, the complexity is

$$
O\left(\sigma^{\left|\alpha_{i}\right|} m \kappa^{m}\right) .
$$

[Case 2] For this case, the contribution of $u_{i}$ to $\operatorname{meu}(S D)$ is also additive, and obtainable from its subnet segment. Formally, in addition to a relation similar to Eq. (12) (except all terms are meu() values), this can be seen from an alternative perspective. Let $\left(\overline{b_{i}}, \overline{y_{i}}\right)$ be a local action profile over $\alpha_{i}$, such that $e u\left(\overline{b_{i}}, \overline{y_{i}}\right)=$ $\max _{\overline{y_{i}}}{ }^{\prime \prime} e u\left(\overline{b_{i}},{\overline{y_{i}}}^{\prime \prime}\right)$, where $e u\left(\alpha_{i}\right)$ is computed from the subnet segment of $u_{i}$. Let $\bar{z}$ over $D \backslash \gamma_{i}$ be a local action profile with $\operatorname{proj}\left(\bar{z}, \beta_{i}\right)=\overline{b_{i}}$, and ${\overline{y_{i}}}^{\prime}$ be a local action profile over $\gamma_{i}$, such that

$$
e u\left(\bar{z}, \bar{y}_{i}^{\prime}\right)=\max _{{\overline{y_{i}}}^{\prime \prime}} e u\left(\bar{z}, \bar{y}_{i}^{\prime \prime}\right),
$$

where $e u(D)$ is computed from $A$ 's subnet. Then, there must be

$$
e u\left(\bar{z},{\overline{y_{i}}}^{\prime}\right)=e u\left(\bar{z}, \overline{y_{i}}\right) .
$$

That is, if a local action profile over private decision ancestors of $u_{i}$ is optimal, viewed from the subnet segment of $u_{i}$, then it is optimal viewed from the entire subnet as well.

Therefore, we can apply PeDecSu to the subnet segment of $u_{i}$. Set parameters of PeDecSu to $D=\alpha_{i}, \beta=\beta_{i}$, and $\gamma=\gamma_{i}$. The return value meu $\left(\beta_{i}\right)$ is the additive contribution of $u_{i}$ to meu $(S D)$. The return value peer $\left(\beta_{i}\right)$ will be used later, and needs to be stored. Applying Proposition 1 to this case, the complexity to obtain $\operatorname{meu}\left(\beta_{i}\right)$ is

$$
O\left(\theta \sigma^{\left|\alpha_{i}\right|} m \kappa^{m}\right)
$$


[Cases 3 and 4] Unlike utility variables in cases 1 and 2, contribution of a case 3 or case 4 utility variable to $m e u(S D)$ cannot be individually and additively evaluated.

Example 16. The contribution of $u_{4}$ in Fig. 10 to meu $\left(d_{2}, d_{3}\right)$ cannot be evaluated independently using its subnet segment, because the segment contains neither $d_{2}$ nor $d_{3}$.

The contribution of $u_{3}$ to $m e u\left(d_{2}, d_{3}\right)$ cannot be evaluated independently using its subnet segment either, because an optimal action profile over $\left\{d_{3}, d_{4}\right\}$ is not necessarily compatible with any optimal action profile over $\left\{d_{3}, d_{4}, d_{5}\right\}$.

We show below that by proper grouping of case 3 and case 4 utility variables into correlated clusters, we can evaluate contribution of each cluster independently and additively. Each cluster is obtained by starting with one variable of case 3, whose existence is established below.

Proposition 4. Let $S D$ be agent interface of a connected subnet, such that there exists a utility variable that is neither case 1 nor case 2, relative to SD. Then there exists at least one utility variable that is case 3.

Proof. We prove by contradiction. Suppose not all utility variables are case 1 or case 2 , and all utilities, that are not case 1 or case 2 , are case 4 . Let $u_{i}$ be such a case 4 variable. By Def. 10, $\alpha_{i}$ is entirely private. Since there exists no case 3 utility variable that shares any decision ancestor in $\alpha_{i}$, it follows that the subnet segment of $u_{i}$ is disconnected from $S D$ : a contradiction to that the subnet is connected. Therefore, from Proposition 3, there must be at least one utility variable that is case 3 .

Proposition 4 says whenever cases 1 and 2 do not cover all utilities, there exist some in case 3 . Let a correlated cluster be initiated with a case 3 utility $u_{i}$. By Def. 10, there exists $u_{j}$ such that $u_{i}$ and $u_{j}$ share a private decision ancestor. Note that $u_{j}$ may be case 3 or case 4 . Add $u_{j}$ to the cluster, and continue until no such utility variable can be found. In Fig. 10, $u_{3}$ and $u_{4}$ form a correlated cluster. Formally, a correlated cluster is defined as follows.

Definition 11. Let $S U=\left\{u_{1}, \ldots, u_{\eta^{\prime}}\right\} \subseteq U$ be a subset of utility variables in a subnet. $S U$ is a correlated cluster if

(1) $u_{1}$ is case 3 ,

(2) for each $u_{i}\left(i=2, \ldots, \eta^{\prime}\right)$, there exists $j<i$ with $\gamma_{j} \cap \gamma_{i} \neq \emptyset$, and

(3) no proper superset of $S U$ satisfies the above two conditions.

We extend subnet segment of a single utility variable to subnet segment of a correlated cluster, which contains utility nodes in the cluster, ancestors of each utility node in the cluster, and arcs among them. The contribution to meu $(S D)$ by utility variables in a correlated cluster can be evaluated independently (of evaluations of 
other clusters) and additively (e.g., Eq. (12)), using its subnet segment. This can be seen by applying the same argument for case 2 to the cluster.

From counter-examples in Example 16, we have Proposition 5 below.

Proposition 5. A correlated utility cluster and its subnet segment is the minimum unit, where contribution of utility variables to meu $(S D)$ can be independently and additively evaluated.

For each correlated cluster with its utility variables indexed as $u_{1}, \ldots, u_{\eta^{\prime}}$, we apply PeDecMu to its subnet segment. Denote $\alpha^{\prime}=\cup_{i=1}^{\eta^{\prime}} \alpha_{i}, \quad \beta^{\prime}=\cup_{i=1}^{\eta^{\prime}} \beta_{i}, \quad \gamma^{\prime}=$ $\cup_{i=1}^{\eta^{\prime}} \gamma_{i}$. Set parameters of PeDecMu to $D=\alpha^{\prime}, \beta=\beta^{\prime}$, and $\gamma=\gamma^{\prime}$. The return value meu $\left(\beta^{\prime}\right)$ is the additive contribution of the cluster to meu $(S D)$. Return value $\operatorname{peer}\left(\beta^{\prime}\right)$ is needed later and is to be stored. Applying Proposition 2, the complexity to obtain $\operatorname{meu}\left(\beta^{\prime}\right)$ is

$$
O\left(\theta \sigma^{\left|\alpha^{\prime}\right|} \eta^{\prime} m \kappa^{m}\right) .
$$

Let $S U_{1}, S U_{2}, \ldots$ be subsets of $U$, where $\cup_{i} S U_{i}=U$, and each $S U_{i}$ is either a singleton under case 1 , or a singleton under case 2 , or a correlated cluster from cases 3 or 4 . Let $\alpha_{i}^{\prime}, \beta_{i}^{\prime}$ and $\gamma_{i}^{\prime}$ denote the sets of decision ancestor variables for $S U_{i}$. Then Eq. (11) can be computed as

$$
u t m_{0}(\overline{s d})=\left(\sum_{i} e u_{i}\left(\operatorname{proj}\left(\overline{s d}, \beta_{i}^{\prime}\right)\right)\right)+\sum_{j} \operatorname{meu}_{j}\left(\operatorname{proj}\left(\overline{s d}, \beta_{j}^{\prime}\right)\right),
$$

where each $e u_{i}()$ is the contribution from a $S U_{i}$ under case 1 , and each $m e u_{j}()$ is the contribution either from a $S U_{j}$ under case 2 or from a $S U_{j}$ under case 3 or 4 .

When $\left|\alpha_{i}^{\prime}\right|$ for each $u_{i}$ under case 1 is small, and there are enough private decision variables in the subnet, the second term in Eq. (16) (involving utility variables of cases 2, 3,4) dominates the computation. From Eqs. (14) and (15), the complexity to obtain $u \mathrm{tm}_{0}(\overline{s d})$ is the following, where $\left|\alpha^{*}\right|=\max _{i}\left|\alpha_{i}^{\prime}\right|$,

$$
O\left(\theta \sigma^{\left|\alpha^{*}\right|} \eta m \kappa^{m}\right) \text {. }
$$

It is significantly more efficient than $O\left(\theta \sigma^{\rho} \eta m \kappa^{m}\right)$ (Proposition 2) as the complexity would be, if PeDecMu is directly applied to the subnet. It is only exponential on cardinality of the largest cluster decision ancestor set, while the latter is exponential on $\rho=|D|$.

\subsection{Utility clusters and messages from general agents}

Before generalizing to an arbitrary agent, we consider non-leaf agents in the first round of message passing. Let $D$ be the set of decision variables of a non-leaf agent $A$. $A$ receives utility messages from child agents $A_{1}, \ldots, A_{k}$, over interfaces $S D_{1}, \ldots, S D_{k}$, respectively, and then computes and sends a utility message over interface $S D$ with parent agent $B$ (see Fig. 11). 


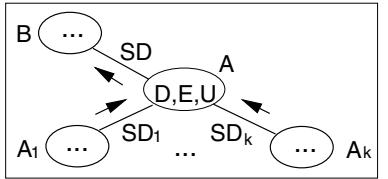

Fig. 11. First round message passing by non-leaf agent $A$

We denote message from $A_{j}$ by $u t m_{j}\left(S D_{j}\right)$, which specifies $u t m_{j}\left(\overline{s d_{j}}\right)$ for each local action profile $\overline{s d_{j}}$ over $S D_{j}$. To absorb incoming $u t m_{j}\left(S D_{j}\right)$ in computing outgoing message $u t m_{0}(S D)$ to $B$, we let $A$ modifies its subnet as follows. The modification is intended to maintain the subnet as regular length-2.

For each decision variable $d_{i} \in S D_{j}$ with domain $O p_{i}$, add a new child node $e_{i}$ with domain $E f_{i}=O p_{i}$. Associate CPT $P\left(e_{i} \mid d_{i}\right)$ with it, such that $P\left(e_{i} \mid d_{i}\right)=$ 1 whenever $e_{i}=d_{i}$ and $P\left(e_{i} \mid d_{i}\right)=0$ otherwise. Hence, $e_{i}$ is deterministically dependent on $d_{i}$. Denote the set of new child nodes added relative to $S D_{j}$ as $S E_{j}$. Add a new utility node $u t m_{j}$ with $S E_{j}$ as its parents, associate it with the function $u t m_{j}\left(S E_{j}\right)$, such that $u t m_{j}\left(S E_{j}\right)=u t m_{j}\left(S D_{j}\right)$, and assign it weight $w_{j}=1$. Note that the weight assignment does not follow sum-to-one stated in Section 2.2. The modification to $A$ 's subnet is summarized in algorithm UpdateSubnet.

After UpdateSubnet, $A$ 's subnet is still regular length-2. Message $u t m_{0}(S D)$ outgoing to $B$ can be computed, using the method in Section 5.1 and Eq. (16). For each $\overline{s d}$ over $S D, u t m_{0}(\overline{s d})$ is the MEU, according to all subnets on hypertree rooted at $A$ 's subnet, as will be seen below.

Algorithm 3. UpdateSubnet

Input: decision subnet $S$ over $D \cup E \cup U$, incoming messages $u t m_{1}\left(S D_{1}\right)$, ..., $u t m_{k}\left(S D_{k}\right)$ over agent interfaces $S D_{1}, \ldots, S D_{k}$, and agent interface $S D$ for outgoing message;

for each $S D_{j}$,

initialize $S E_{j}=\emptyset$;

for each $d_{i} \in S D_{j}$,

add child node $e_{i}$ to $d_{i}$ in $S$

set domain of $e_{i}$ to $E f_{i}=O p_{i}$;

set CPT of $e_{i}$ to deterministic $P\left(e_{i} \mid d_{i}\right)$;

$S E_{j}=S E_{j} \cup\left\{e_{i}\right\}$

add utility node $u t m_{j}$ in $S$;

for each $e \in S E_{j}$, connect $e$ as a parent of $u t m_{j}$;

for each $d_{i} \in S D_{j}$, rename variable $d_{i}$ in $u t m_{j}\left(S D_{j}\right)$ as $e_{i}$;

associate function $u t m_{j}\left(S E_{j}\right)$ from last step with node $u t m_{j}$;

set weight for node $u t m_{j}$ to $w_{j}=1$;

return;

For the root agent, after UpdateSubnet, it performs PeDecMu with $\beta=\emptyset$ to 
obtain an optimal local action profile $\bar{d}^{*}$. The first round of message passing now ends.

Algorithm 4. CollectUtilPeUc

Input: decision subnet of agent $A$;

1 if $A$ is not a leaf agent,

for each child agent $A_{i}$, receive $u t m_{j}\left(S D_{j}\right)$;

call UpdateSubnet;

if $A$ is root agent, call $\mathrm{PeDecMu}$ with $\beta=\emptyset$ and get return value $\bar{d}^{*}$;

return $\bar{d}^{*}$;

denote the set of utility variables in current subnet by $U$;

$U^{\prime}=U$

9 classify variables in $U^{\prime}$ into 4 cases;

10 while $U^{\prime} \neq \emptyset$,

11 remove $u_{i} \in U^{\prime}$ from $U^{\prime}$;

12 if $u_{i}$ is case 1 , compute $e u\left(\alpha_{i}\right)$ using subnet segment of $u_{i}$ and Eq. (3);

13 else if $u_{i}$ is case 2 ,

14 call PeDecSu with subnet segment of $u_{i}$ and parameters $\alpha_{i}, \beta_{i}, \gamma_{i}$;

15 get return value $\left(\operatorname{meu}\left(\beta_{i}\right)\right.$, peer $\left.\left(\beta_{i}\right)\right)$ and save $\operatorname{peer}\left(\beta_{i}\right)$;

16 else if $u_{i}$ is case 3 ,

17 for each $u_{j} \in U^{\prime}$ in the same correlated cluster with $u_{i}$, remove $u_{j}$ from $U^{\prime}$;

18 call PeDecMu with subnet segment of the cluster and $\alpha^{\prime}, \beta^{\prime}, \gamma^{\prime}$;

19 get return value $\left(\operatorname{meu}\left(\beta^{\prime}\right)\right.$, peer $\left.\left(\beta^{\prime}\right)\right)$ and save $\operatorname{peer}\left(\beta^{\prime}\right)$;

20 compute $u t m_{0}(S D)$ by Eq. (16) from eu( $\left.\alpha_{i}\right), \operatorname{meu}\left(\beta_{i}\right)$ and meu $\left(\beta^{\prime}\right)$ above;

21 send $u t m_{0}(S D)$ to agent $B$;

Algorithm 4 specifies the overall operation of a general agent $A$, during the first round of message passing, whose parent agent, if any, is $B$, and whose child agents, if any, are $A_{1}, \ldots, A_{k}$. The interface of $A$ with $B$ is $S D$, and that with $A_{i}$ is $S D_{i}$.

A leaf agent executes lines 7 through 21 . The root agent executes lines 1 through 6 . Each other agent executes all lines except 4 through 6 . For such an agent, the subnet at line 7 is the updated one and so is the set $U$.

Proposition 6 establishes the key property of CollectUtilPeUc, when executed by non-root agents.

Proposition 6. For each non-root agent A, after running CollectUtilPeUc, its message utm ${ }_{0}(S D)$ is the $M E U$ function with respect to partial action profiles, over union of subsystems on the sub-hypertree rooted at $A$.

Proof. We prove by induction on depth $\operatorname{dep} \geq 1$ of the hypertree (length of the longest path from the root to a leaf). When $\operatorname{dep}=1, A$ is a leaf, and $u t m_{0}(S D)$ is obtained by Eq. (16). From Propositions 1 and 2, the statement holds. 
Assume that the statement holds for $d e p \leq k$, where $k \geq 1$. Consider $d e p=k+1$. $A$ is non-leaf, and lines 2 and 3 are run. Due to the inductive assumption, during UpdateSubnet, adding subnet segment for each incoming message $u t m_{j}\left(S D_{j}\right)$ is equivalent to including in Eq. (16) a MEU function for the sub-hypertree rooted at child agent $A_{j}$. Hence, $u t m_{0}(S D)$ is the MEU function for the sub-hypertree rooted at $A$.

Proposition 7 establishes the key property of CollectUtilPeUc, when executed by the root agent.

Proposition 7. For the root agent A, after running CollectUtilPeUc, the return local action profile $\bar{d}^{*}$ is globally optimal.

Proof. Agent $A$ runs only lines 1 through 6 . By an inductive argument similar to the proof of Proposition 6, note that during UpdateSubnet, adding subnet segment for each incoming message $u t m_{j}\left(S D_{j}\right)$ is equivalent to including in Eq. (7) a MEU function for the sub-hypertree rooted at child agent $A_{j}$. From Proposition 2, the statement holds.

\subsection{Decision message distribution}

The second round of message passing starts at the root agent. It projects the optimal local action profile to interface with each adjacent agent on hypertree, and sends the projected action profile to the agent. When a non-root agent $A$ receives the local action profile $\overline{s d}^{*}$ over its interface $S D$ with the parent agent $B$, it uses the message to compute its optimal local plan. The computation is organized based on the four case classification of its utility variables (Section 5.1).

If $u_{i}$ is case 1 , then $\alpha_{i} \subset S D$, and the optimal local action profile over $\alpha_{i}$ is

$$
\overline{s d}_{i}^{*}=\operatorname{proj}\left(\overline{s d}^{*}, \alpha_{i}\right) \text {. }
$$

If $u_{i}$ is case $2, A$ obtains ${\overline{b_{i}}}^{*}=\operatorname{proj}\left(\overline{s d}^{*}, \beta_{i}\right)$, and retrieves ${\overline{y_{i}}}^{*}=\operatorname{peer}\left({\overline{b_{i}}}^{*}\right)$ using the peer function, stored during CollectUtilPeUc. The optimal local action profile over $\alpha_{i}$ is

$$
{\overline{s d_{i}}}^{*}=\left({\overline{b_{i}}}^{*},{\overline{y_{i}}}^{*}\right) .
$$

For case 3 and 4 utility variables in the same correlated cluster (which we index by $i$ ), the optimal plan over the cluster's decision ancestor set $\alpha^{\prime}$ is obtained. $A$ obtains $\bar{b}^{\prime *}=\operatorname{proj}\left(\overline{s d}^{*}, \beta^{\prime}\right)$, and retrieves $\bar{y}^{\prime *}=\operatorname{peer}\left(\bar{b}^{\prime *}\right)$ using the peer function, stored during CollectUtilPeUc. The optimal local action profile over $\alpha^{\prime}$ is

$$
{\overline{s d_{i}}}^{*}=\left(\bar{b}^{\prime *}, \bar{y}^{\prime *}\right) \text {. }
$$

After the optimal local action profile over each $\alpha_{i}$ (cases 1 and 2) or $\alpha^{\prime}$ (cases 3 and 4 ) is specified, the optimal local action profile over $D$ (decision nodes in $A$ ) is the action profile join

$$
\bar{d}^{*}=\left({\overline{s d_{1}}}^{*},{\overline{s d_{2}}}^{*}, \ldots\right)
$$


Algorithm DistributeActionPeUc specifies operations of a general agent $A$.

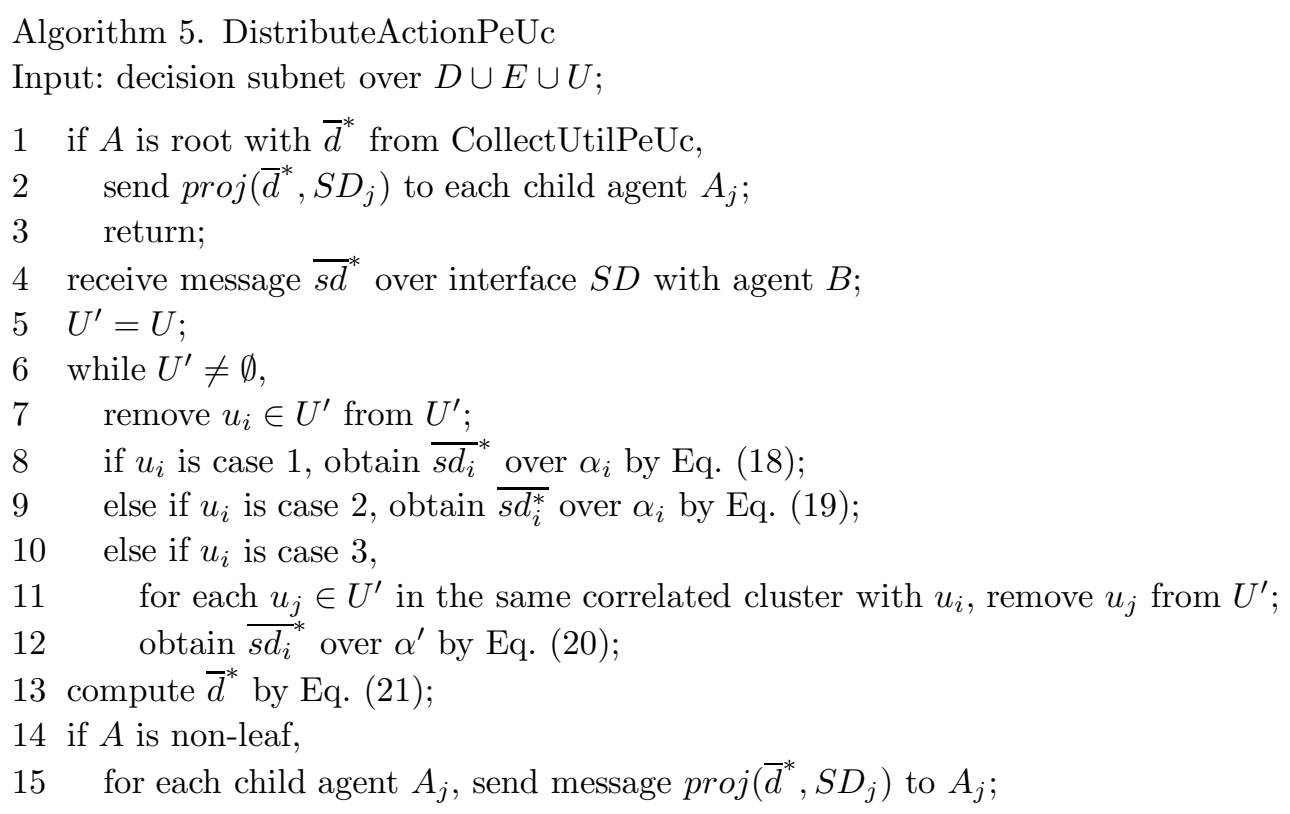

The root agent executes lines 1 through 3 . A leaf agent executes lines 4 through 13. Each other agent executes lines 4 through 15 . The second round of message passing ends at leaf agents. The system coordinator executes DecisionPeUc, which causes distributed execution of the above algorithms.

Algorithm 6. DecisionPeUc

select an agent $A$ arbitrarily;

call CollectUtilPeUc in $A$;

call DistributeActionPeUc in $A$;

Theorem 1 establishes the optimality of DecisionPeUc. The joint action profile in the theorem is a virtual object, and is never physically constructed.

Theorem 1. After DecisionPeUc, the joint action profile formed by joining the local action profile $\bar{d}^{*}$ at each agent is optimal.

Proof. By Proposition 7, after the root agent $A$ completes CollectUtilPeUc, $\bar{d}^{*}$ obtained is the projection of a globally optimal joint action profile to the root subsystem.

The optimal local action profile under each interface constraint is stored as a peer () function, in CollectUtilPeUc. It is retrieved in DistributeActionPeUc by each child agent $A_{j}$ of root. Hence, $\bar{d}^{*}$ obtained by $A_{j}$, during DistributeActionPeUc, is the projection of the globally optimal joint action profile to the subsystem at $A_{j}$. Applying this argument recursively for each non-root agent down the hypertree, $\bar{d}^{*}$. 
obtained by the agent during DistributeActionPeUc is the projection of the globally optimal joint action profile to the subsystem of the agent.

From Eq. (17), the total complexity of DecisionPeUc is approximately

$$
O\left(n \theta \sigma^{\left|\alpha^{*}\right|} \eta m \kappa^{m}\right) .
$$

This represents a complexity reduction by a ratio of $\theta / \sigma^{\rho-\left|\alpha^{*}\right|}$, relative to the earlier algorithm. ${ }^{15}$ It consists of an exponential factor $\sigma^{\rho-\left|\alpha^{*}\right|}$ due to utility clustering, as well as a linear factor $\theta$ due to partial evaluation.

\section{Experimental Evaluation}

To evaluate the effectiveness of DecisionPeUc empirically, multiple sets of experiments were performed. We report two sets of them that are representative. The first set evaluates efficiency gain due to partial evaluation and utility clustering, both individually and in combination. The experiments confirm the linear efficiency gain due to partial evaluation, and the exponential improvement due to utility clustering. Section 6.1 reports setup and result for this set of experiments.

The second set assesses the robustness of the pivot assumption (Def. 9). The pivot assumption states that, for each effect variable, probabilities of pivot effects for different action profiles are identical. The first set of experiments above also confirms optimal decision making under pivot assumption. The second set of experiments shows that optimal or near-optimal decision making can be achieved when the assumption is relaxed. Setup and result of this set of experiments are reported in Section 6.2.

\subsection{Efficiency gain due to partial evaluation and utility clustering}

MAE is used as the testbed (Example 3 with $T=1$ ). The multiagent system, referred to by $4 \mathrm{~A} 6 \mathrm{D}$, consists of $n=4$ agents, with the hypertree organization $A_{1}-A_{2}-A_{3}-A_{4}$. Agent subnets are structured similarly to those in Example 3, and are converted equivalently to regular length-2. Each agent has between two to three movement decisions that are shared with agents adjacent on the hypertree. In addition, each agent has 6 private decisions. Each decision variable has domain cardinality $\sigma=5$. Each effect variable has domain cardinality $\kappa=5$.

The $4 \mathrm{~A} 6 \mathrm{D}$ agent team is placed at 30 distinct team locations, and simultaneous decision making is performed for each team location, to determine the optimal joint action profile. For each team location, uncertainty on movements and other actions is represented in agent subnet CPTs, and reward distribution at neighborhood cells is encoded by utility functions. For all CPTs, the pivot assumption holds exactly, with the pivot probability value being 0.9 . All utility functions are such that the density of high utility cells (of utility value $>0.5$, where each utility $\in[0,1]$ ) is about $5 \%$. 
The maximum number of shared decisions per agent is 3 , and the number of private decisions per agent is 6 . Hence, the maximum number of decisions per agent is 9 . The corresponding number of local action profiles per agent is $1,953,125$. The number of joint action profiles for $4 \mathrm{~A} 6 \mathrm{D}$ team is $1.455 \times 10^{25}$.

Four alternative methods for simultaneous decision making in CDNs are implemented distributively in Java:

FeNoUc The existing method ${ }^{15}$ that is based on full evaluation

FeUc The existing method enhanced by utility clustering

PeNoUc The existing method enhanced by partial evaluation

PeUc The algorithm DecisionPeUc

Simultaneous decision making is run, distributively using a dual-core $2.9 \mathrm{GHz}$ laptop, for each team location, by each method, with $A_{4}$ as the root agent.

For each team location, the decision made by FeNoUc is used as the golden standard, since it is proven to be optimal, is based on full evaluation (not influenced by pivot assumption), and is not influenced by utility clustering. The expected utility of the joint action profile obtained by FeNoUc is then compared against those obtained by alternative methods. For each team location, each of the other three methods returned joint action profiles with the expected utility identical to that of FeNoUc. Hence, optimal simultaneous decision making is achieved by all methods in each case. This confirms the optimality of DecisionPeUc, under the pivot assumption.

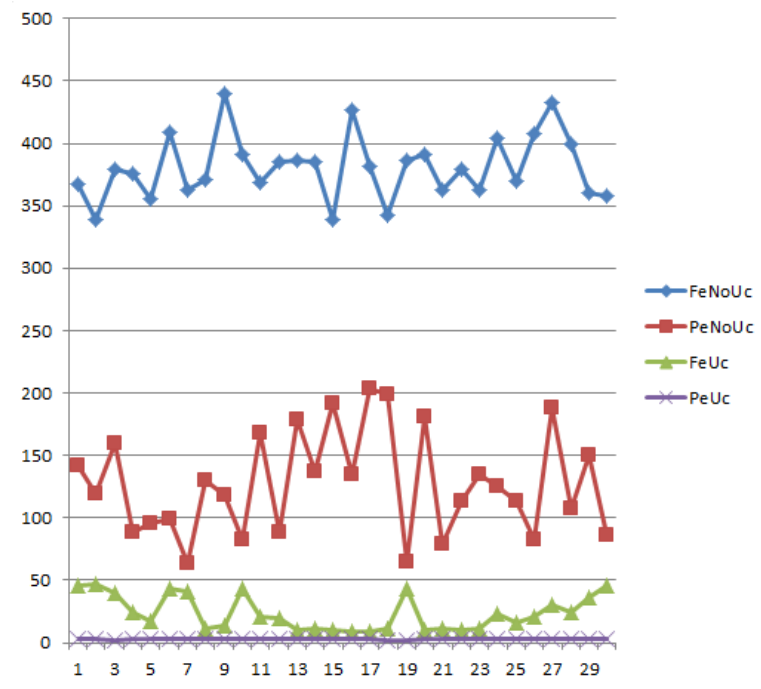

Fig. 12. 4A6D team runtimes by four alternative methods. The $\mathrm{x}$-axis is indexed by team location, and the $\mathrm{y}$-axis measures runtime in seconds. 
Fig. 12 depicts team runtime for each team location by each method. The mean team runtime for each method across all team locations, and the corresponding standard deviation are summarized in Table 5 .

Table 5. Summary of runtimes (in seconds) by alternative methods

\begin{tabular}{|l|rrrr|}
\hline \hline & FeNoUc & PeNoUc & FeUc & PeUc \\
\hline mean & 380.8 & 128.6 & 23.8 & 2.8 \\
stdev & 25.6 & 41.4 & 13.9 & 0.3 \\
\hline \hline
\end{tabular}

The impact of partial evaluation on efficiency can be observed by comparing runtimes between FeNoUc and PeNoUc for each team location, and averaging the ratios. The comparison can also be performed between FeUc and PeUc. The result from data plotted in Fig. 12 shows that PeNoUc runs about 3.3 times as fast as FeNoUc, and PeUc runs about 8.7 times as fast as FeUc. In both pairs of comparisons, the runtime improvement reflects the percentage of action profiles that are fully evaluated: a linear factor. We observe that partial evaluation works more effectively, by a ratio of $2.6(8.7 / 3.3)$, when coupled with utility clustering.

The impact of utility clustering on efficiency can be observed by a similar averaging of runtime ratio, between FeNoUc and FeUc, and between PeNoUc and PeUc. The result from data plotted in Fig. 12 shows that, FeUc runs about 22.4 times as fast as FeNoUc, and PeUc runs about 46.5 times as fast as PeNoUc. The largest utility cluster in a 4A6D agent contains 6 decision variables, and the corresponding subnet has 9 decision variables, each of which has 5 possible actions. Hence, the theoretical exponential factor of efficiency gain by utility clustering is $5^{9-6}=125$ times (see end of Section 5.3). We observe that utility clustering works more effectively, by a factor of $2.1(46.5 / 22.4)$, when it is coupled with partial evaluation.

The combined impact of partial evaluation and utility clustering on efficiency can be evaluated by comparing runtimes between FeNoUc and PeUc. The data shows that PeUc runs about 138.7 times as fast as FeNoUc: combining a linear factor and an exponential.

The statistical significance of the above comparisons are verified with Friedman test. The rank sums of FeNoUc, PeNoUc, FeUc, and PeUc are 120, 90 60, 30, respectively, resulting in the test statistics 90 . For $\alpha=0.005$, the critical value is 12.84. Hence, null hypothesis is rejected at the $\alpha=0.005$ level of significance. For post-hoc analysis, 3 pairwise comparisons are performed, FeNoUc versus PeNoUc, PeNoUc versus FeUc, and FeUc versus PeUc. The rank sum difference between each pair is 30 , that is larger than the critical value 29.35 for $\alpha=0.005$. Hence, the null hypothesis in the one-sided test is rejected for each pair. In other words, at the $\alpha=0.005$ level, PeUc is significantly faster than FeUc, FeUc is significantly faster than PeNoUc, and PeNoUc is significantly faster than FeNoUc. 
Beside the above reported, we have run several additional sets of experiments, including teams $4 \mathrm{~A} 3 \mathrm{D}, 4 \mathrm{~A} 4 \mathrm{D}$, and 4A5D (with 3,4 and 5 private decisions per agent, respectively). The results generally demonstrate the similar trends, as reported above. Due to difference in numbers of decision variables, runtimes by $\mathrm{Fe}-$ NoUc increase by roughly 5 times among 4A3D, 4A4D, 4A5D, 4A6D, in that order. As decisions by 4A3D, 4A4D, 4A5D teams are less expensive, runtime differences between FeUc and PeUc are not as pronounced as 4A6D reported above (PeUc already takes only $2.8 \mathrm{~s}$ ), due to computation overhead not counted in the complexity analysis. Since results from teams such as 4A3D, 4A4D, 4A5D do not reveal as well, as the results from $4 \mathrm{~A} 6 \mathrm{D}$, the general trends for scaling up, they are not included in our report. On the other hand, more expensive teams, such as $4 \mathrm{~A} 7 \mathrm{D}$, would take significantly longer to run (at least $380 \mathrm{~s} \times 5=1900 \mathrm{~s}$ per execution of $\mathrm{FeNoUc}$ ), but are expected to show only similar trends as $4 \mathrm{~A} 6 \mathrm{D}$, they are not tested in large scale.

To evaluate how well PeUc scales up, we extended $4 A 6 D$ agent team into additional agent teams $6 \mathrm{~A} 6 \mathrm{D}, 8 \mathrm{~A} 6 \mathrm{D}, 10 \mathrm{~A} 6 \mathrm{D}, 12 \mathrm{~A} 6 \mathrm{D}, 14 \mathrm{~A} 6 \mathrm{D}, 16 \mathrm{~A} 6 \mathrm{D}, 18 \mathrm{~A} 6 \mathrm{D}, 20 \mathrm{~A} 6 \mathrm{D}$ and have run $\mathrm{PeUc}$ on each. That is, each team has $n=4,6,8,10,12,14,16,18,20$ agents. Each agent has up to 3 shared decisions and 6 private decisions. Recall that the number of joint action profiles for $4 \mathrm{~A} 6 \mathrm{D}$ team is $1.455 \times 10^{25}$. Each additional agent increases that number by 78,125 times. Fig. 13 depicts their runtime in seconds. As $n$ scales up from 4 to 20, the runtime grows slightly more than linear. Although hyperchain agent organizations are used, the result is representative for hypertrees of radius $n$ (with the root agent at the center). This demonstrates that PeUc scales up well. For 20 agents, it takes about 1 minute and hence near real-time performance is achieved by $20 \mathrm{~A} 6 \mathrm{D}$ team.

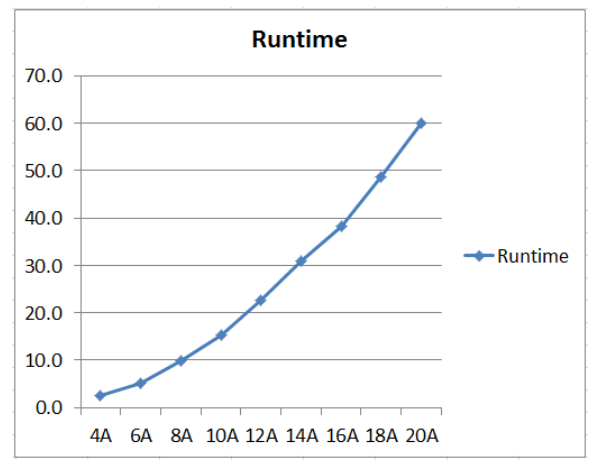

Fig. 13. PeUc runtime for teams 4A6D, 6A6D, 8A6D, 10A6D, 12A6D, 14A6D, 16A6D, 18A6D, and $20 \mathrm{~A} 6 \mathrm{D}$ 


\subsection{Sensitivity of optimality to pivot assumption}

The pivot assumption requires that, for each effect variable, probabilities of pivot effects for different action profiles of its decision parents are identical. The first set of experiments reported above confirmed optimality of DecisionPeUc, when the assumption holds exactly. Since the assumption cannot be expected to hold for every application, we report below a second set of experiments, to assess robustness of the pivot assumption.

Let $e_{i}$ be an effect variable of 5 possible values (the case in our testbed), with decision parent set $\delta_{i}$. The pivot probability for any action profile over $\delta_{i}$ must be in the range $[0.2,1)$. The lower bound 0.2 corresponds to a uniform distribution, and the upper bound to the case that all possible values of $e_{i}$ are very unlikely, except the pivot effect. This range constrains our setup on pivot probabilities.

The set of experiments are divided into three groups. Each group consists of 30 4A3D team locations. For the first group, all CPTs in agent subnets have pivot probabilities in the range of $[0.8,0.9]$. The upper bound 0.9 is identical to the pivot probability in the first set of experiments. For instance, if pivot probabilities in Table 1 were in the range of $[0.8,0.9]$, then at least one row would have the pivot probability 0.9 , at least one row would have 0.8 , and other rows would have pivot probabilities in between. With pivot probabilities scattered between 0.8 and 0.9 for different action profiles, the pivot assumption no longer hold. The range for the second group is $[0.7,0.9]$, which is twice the width as the first group. The range for the third group is $[0.3,0.9]$, which covers almost the whole constraint range $[0.2,1)$. A total of 90 team locations are used in the experiments.

To adopt PeUc to these environments, the method works as if the pivot assumption holds, but the pivot probability value used in decision making is either the maximum or the minimum pivot probability value in the CPT. We refer to the modified methods as PeUcMax and PeUcMin, respectively. For example, for the first group above, PeUcMax uses 0.9 as the pivot probability, and PeUcMin uses 0.8 .

For each of the 90 team locations, we run FeUc to obtain the optimal expected utility over joint action profiles, as it does not depend on partial evaluation, is exact, and is more efficient than FeNoUc. We then run PeUcMax and PeUcMin, and calculate the ratio between the expected utility of the decision they obtain, and that of the decision obtained by FeUc. We will refer to this ratio as the optimality ratio (OR). If an execution of PeUcMax or PeUcMin obtains the identical expected utility as FeUc, the optimality ratio is 100 percent. A suboptimal result is indicated by a less than 100 percent ratio.

Fig. 14 (left) depicts the optimality ratios from PeUcMax, and Fig. 14 (right) depicts those from PeUcMin. The number of suboptimal runs, the mean optimality ratio, and the standard deviation, for each group and each method, are summarized in Table 6.

As shown by Table 6 (2nd and 3rd column), for pivot probability (PP) range 
$[0.8,0.9]$, both PeUcMax and PeUcMin made optimal decisions, for each of the 30 cases in the first group. It demonstrates that optimal decision is still achievable, when the pivot assumption does not hold, as long as pivot probability values are reasonably close.

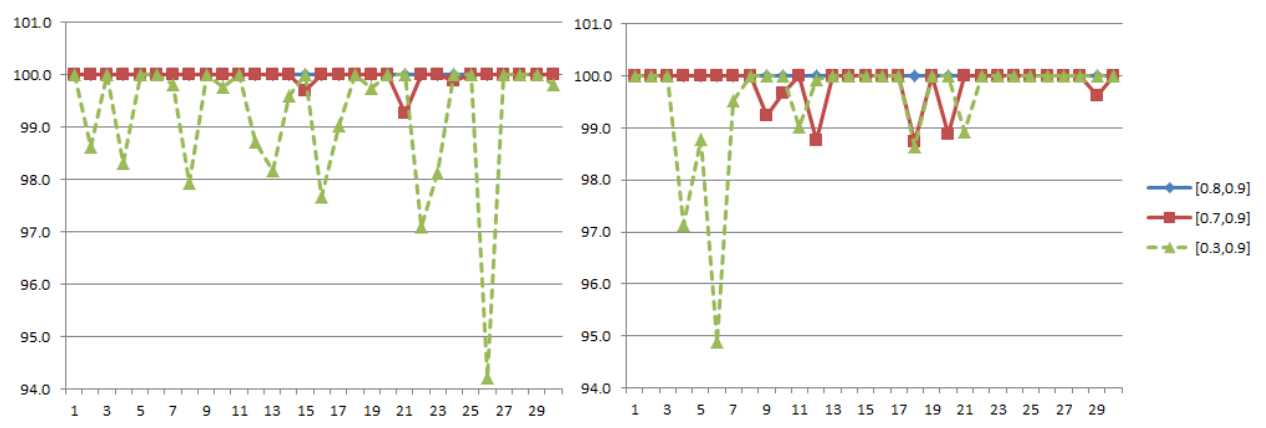

Fig. 14. Left: Optimality ratios from PeUcMax. Right: Optimality ratios from PeUcMin.

Table 6. Summary on numbers of suboptimal runs and optimality ratios by PeUcMax and PeUcMin

\begin{tabular}{|c|rr|rr|rr|}
\hline \hline PP range & \multicolumn{2}{|c|}{$[0.8,0.9]$} & \multicolumn{2}{|c|}{$[0.7,0.9]$} & \multicolumn{2}{|c|}{$[0.3,0.9]$} \\
\hline & PeUcMax & PeUcMin & PeUcMax & PeUcMin & PeUcMax & PeUcMin \\
\hline subop runs & 0 & 0 & 3 & 6 & 15 & 8 \\
mean $O R$ & 100 & 100 & 99.96 & 99.8 & 99.2 & 99.6 \\
stdev OR & 0 & 0 & 0.1 & 0.4 & 1.3 & 1.1 \\
\hline \hline
\end{tabular}

As shown by 4 th and 5 th column, when pivot probability range widens to $[0.7,0.9]$, between $10 \%$ ( 3 out of 30 by PeUcMax) and $20 \%$ ( 6 out of 30 by PeUcMin) of decisions are suboptimal. However, these suboptimal decisions yield no less than $98.5 \%$ of the optimal expected utility (see Fig. 14). It demonstrates that near optimal decision is achievable, when the pivot assumption is clearly violated, and pivot probability values are fairly different.

When the pivot probability range widens to $[0.3,0.9]$ in the third group (last two columns), it represents the worst condition, as far as the violation of the pivot assumption is concerned. The percentages of suboptimal decisions reach $50 \%$ by PeUcMax and $27 \%$ by PeUcMin. Still, no less than $94 \%$ of the optimal expected utility is obtained.

Overall, the experiments demonstrate that, although pivot assumption is sufficient for optimality of partial evaluation, it is not a necessary condition. Partial evaluation is fairly robust with respect to the pivot assumption. Hence, combined with utility clustering, DecisionPeUc is widely applicable, where pivot assumption does not hold exactly, and it enables optimal or near-optimal simultaneous decision 
making with significant efficiency gain.

\section{Related Work}

We discuss several frameworks for cooperative multiagent decision making. It is not an exhaustive review, but to note literature that are most closely related to CDNs. Where a framework is primarily for competitive multiagent, it is indicated explicitly.

\subsection{Decision-theoretic frameworks}

A number of frameworks for sequential decision making have been developed based on decentralized partially observable Markov decision process (DEC-POMDP) and its fully observable counterpart (DEC-MDP). The problem of generating optimal policies for DEC-POMDPs is known to be NEXP-complete. ${ }^{3}$ CDNs focus on simultaneous decision making. The complexity of optimal decision making in general CDNs is exponential on the number of decision variables over all agents. ${ }^{15}$ Hence, optimal solution methods are intractable for general DEC-MDPs ${ }^{2}$ and DECPOMDPs, ${ }^{17}$ as well as for general CDNs.

Transition and observation independence (TOI) has been exploited for more tractable optimal policy generation ${ }^{18-20}$ in TOI DEC-POMDPs. TOI implies that actions taken by one agent cannot affect any other agent's observation or local state. Because decision variables in CDN agent interfaces affect local states at other agents, systems encoded as CDNs are not subject to TOI restriction.

Policies for DEC-MDPs or DEC-POMDPs can be generated offline at planning time. Generation can be conducted centrally ${ }^{17,18,21,22}$ or distributively. ${ }^{23}$ Alternatively, planning can be performed online ${ }^{24,25}$ to determine the next joint action profile. Since only the actual observation history, rather than all possible histories, needs to be considered, the planning can be more efficient. Between the two alternatives, execution-centric frameworks ${ }^{21,26,27}$ combine simplified offline planning with execution-time communication of agent observation-action sequence to achieve coordination. CDNs focus primarily on simultaneous decision making. However, they are applicable to some Dec-POMDPs such as MAE ( see $^{16}$ for relation between MAE and Dec-POMDP), where computation in CDNs is similar to online planning (see discussion after Example 2). DEC-POMDPs online planning methods ${ }^{24,25}$ are approximate, while decision making methods for $\mathrm{CDNs}^{15}$ and this work) are optimal.

A DEC-POMDP environment is described by a set of states. ${ }^{3,17,21,23,26} \mathrm{~A}$ factored DEC-POMDP has a state space spanned by a set of state variables, ${ }^{2,22}$ and allows conditional independence among variables to be exploited by encoding transition and observation models through graphical models. It also allows decomposition of reward model by exploiting additive or other independence.

MSBNs, evolved from their modular forms ${ }^{10,14}$ in early 1990 s to multiagent, ${ }^{11,28}$ are among the earliest multiagent probabilistic graphical models. CDNs are extensions of MSBNs from probabilistic frameworks to decision theoretic ones. Both 
MSBNs and CDNs employ the hypertree decomposition (Def. 1). The hypertree decomposition is explored subsequently in other probabilistic graphical models, e.g., OOBNs $^{12}$ and some Dec-MDP frameworks. ${ }^{13}$

In addition to the hypertree decomposition, MSBNs also pioneer in inter-agent message decomposition, through linkage trees. ${ }^{14}$ Neither OOBNs, nor Dec-MDP frameworks, ${ }^{13}$ nor the earlier CDN framework ${ }^{15}$ explore such message decomposition. A main contribution of the current work is to enable exploration of inter-agent message decomposition in CDNs, through utility clusters (Section 5). Note also that planning in some Dec-MDP frameworks ${ }^{13}$ is approximate, while decision making in CDNs is optimal.

Some frameworks for DEC-MDP ${ }^{2}$ or DEC-POMDP,${ }^{26}$ assume that models are common knowledge. Other frameworks use centralized offline planning. ${ }^{17,18,21,22,27}$ These frameworks are not concerned with agent privacy. In CDN decision making, identities of private variables, their associated numerical information, and decisions made over private decision variables are neither centralized nor communicated. Hence, simultaneous decision making in CDNs preserves agent privacy. Such privacy preservation is necessary for certain applications, e.g., collaborative industrial design, ${ }^{8}$ and CDNs are well positioned to support them.

$\mathrm{IDs}^{29}$ can be viewed as a subclass of factored POMDPs. IDs support sequential decision making, while CDNs focus on simultaneous decision making. CDN subnets differ from IDs as detailed in Section 2.3. IDs allow chance parents for decision nodes. Such is not needed, and is disallowed in CDN subnets. Arcs between decision nodes in IDs are not associated with quantitative knowledge. In CDN subnets, such arcs are associated with decision constraints, specified in terms of CPTs.

CDNs differ significantly from MAIDs. ${ }^{4}$ An MAID is a centralized representation of a game among competitive agents. The developer of an MAID oversees the payoff structures of all agents, has the access of the entire MAID, and can analyze it by centralized computation. A CDN is a distributed representation of cooperative agents (with privacy) for optimal decision making. Each CDN agent has the access of its own subnet, without any knowledge about private variables of other subnets, nor their internal structures and numerical parameters. There is no agent in the system, who has the knowledge over all subnets. An optimal joint action profile emerges from distributed simultaneous decision making (Theorem 1). It cannot be derived by centralized computation, since no agent has all the knowledge necessary, nor is it physically represented centrally anywhere.

\subsection{Other related frameworks}

A widely applied multiagent framework is BDI, ${ }^{1,30}$ which models the informational, motivational, and deliberative states of an agent in terms of belief, desire, and intention, respectively. The framework is not limited to cooperative agents. BDI is primarily a logic framework, and it has no quantitative evaluation of degree of optimality. On the other hand, CDN is decision-theoretic with probabilistic belief 
representation, and utility based preference quantification.

Distributed constraint optimization (DCOP) is another area of multiagent cooperation. DCOP problems do not typically involve stochastic environments and hence do not consider uncertainty. A number of frameworks for solving DCOP problems exist, and an extensive review of them is beyond scope of this work. One particular framework, however, based on DPOP ${ }^{5}$ and its various extensions, ${ }^{31}$ is closely related to CDN decision making methods. Computation in both DPOP and CDNs follow a dynamic-programming style. However, CDN models uncertainty, while DPOP does not. DPOP uses a pseudo-tree organization, while CDN uses a junction tree organization. An organizational unit in DPOP is a single-variable-agent, while a unit in $\mathrm{CDN}$ is a multi-variable-subsystem.

\section{Conclusion}

This work makes a number of contributions to simultaneous decision making for cooperative multiagent systems in stochastic environment.

First, the agent interface in CDNs is extended to include certain chance variables while maintaining its support to more efficient decision making. Second, equivalent representation and transformation of CDNs into regular length-2 CDNs are developed, which facilitates model manipulation during decision making. The third contribution is partial evaluation, sanctioned by the pivot assumption, which ensures optimal decision making while improving efficiency by a linear factor. The experimental evaluation shows that partial evaluation is robust with respect to the pivot assumption, and is thus widely applicable. In particular, a sufficiently wide range of pivot probabilities enables the optimal or near optimal partial evaluation. Fourth, subnet decomposition by utility clusters is developed, sanctioned by existence of private decisions in each agent. Utility clustering improves efficiency further by an exponential factor. Finally, a new, general simultaneous decision making algorithm suite is formulated, that integrates the above techniques.

A number of directions deserve further investigation, including a characterization of length-2 equivalent CDNs, a general algorithm of decision equivalent transformation of CDNs into regular length-2, a necessary and sufficient condition for optimal partial evaluation, and a time-bounded partial evaluation for time-critical applications.

\section{Acknowledgements}

Financial support from NSERC, Canada through Discovery Grant is acknowledged.

\section{References}

1. A. Rao and M. Georgeff, "BDI agents: from theory to practice", Proc. 1st Inter. Conf. on Multi-Agent Systems, 1995, pp. 312-319.

2. C. Boutilier, "Planning, learning and coordination in multiagent decision processes", Proc. Conf. on Theoretical Aspects of Rationality and Knowledge, 1996, pp. 195-210. 
3. D.S. Bernstein, S. Zilberstein, and N. Immerman, "The complexity of decentralized control of Markov decision processes", Proc. 16th Conf. on Uncertainty in Artificial Intelligence, 2000, pp. 32-37.

4. D. Koller and B. Milch, "Multi-agent influence diagrams for representing and solving games", Proc. 17th Inter. Joint Conf. on Artificial Intelligence, 2001, pp. 1027-1034.

5. A. Petcu and B. Faltings, "A scalable method for multiagent constraint optimization", Proc. 19th Inter. Joint Conf. on Artificial Intelligence, 2005, pp. 266-271.

6. T. Leaute and B. Faltings, "Protecting privacy through fistributed vomputation in multi-agent decision making", J. Artificial Intelligence Research. 47 (2013) 649-695.

7. Y. Xiang, Y. Mohamed, and W. Zhang, "Distributed constraint satisfaction with multiply sectioned constraint networks", International J. Information and Decision Sciences. 6(2) (2014) 127-152.

8. Y. Xiang, J. Chen, and A. Deshmukh, "A decision-theoretic graphical model for collaborative design on supply chains", in Advances in Artificial Intelligence, LNAI 3060, eds. A.Y. Tawfik and S.D. Goodwin (Springer, 2004) pp. 355-369.

9. J. Pearl, Probabilistic Reasoning in Intelligent Systems: Networks of Plausible Inference (Morgan Kaufmann, 1988).

10. Y. Xiang, D. Poole, and M. Beddoes, "Exploring localization in Bayesian networks for large expert systems", Proc. 8th Conf. on Uncertainty in Artificial Intelligence, 1992, pp. 344-351.

11. Y. Xiang, "Distributed multi-agent probabilistic reasoning with Bayesian networks", in Methodologies for Intelligent Systems, eds. Z.W. Ras and M. Zemankova (SpringerVerlag, 1994) pp. 285-294.

12. D. Koller and A. Pfeffer, "Object-oriented Bayesian networks", Proc. 13th Conf. on Uncertainty in Artificial Intelligence, 1997, pp. 302-313.

13. C. Guestrin and G. Gordon, "Distributed planning in hierarchical factored MDPs", Proc. 18th Conf. on Uncertainty in Artificial Intelligence, 2002, pp. 197-206.

14. Y. Xiang, D. Poole, and M. Beddoes, "Multiply sectioned Bayesian networks and junction forests for large knowledge based systems", Computational Intelligence. 9(2) (1993) 171-220.

15. Y. Xiang, J. Chen, and W.S. Havens, "Optimal design in collaborative design network", Proc. 4th Inter. Joint Conf. on Autonomous Agents and Multiagent Systems (AAMAS'05), 2005, pp. 241-248.

16. Y. Xiang and F. Hanshar, "Multiagent expedition with graphical models", Inter. J. Uncertainty, Fuzziness and Knowledge-Based Systems. 19(6) (2011) 939-976.

17. M.T.J. Spaan, F.A. Oliehoek, and C. Amato, "Scaling up optimal heuristic search in Dec-POMDPs via incremental expansion", Proc. 6th Workshop on Multiagent Sequential Decision-Making in Uncertain Domains, 2011, pp. 63-70.

18. R. Becker, S. Zilberstein, V. Lesser, and C.V. Goldman, "Solving transition independent decentralized Markov decision processes", J. Artificial Intelligence Research. 22 (2004) 423-455.

19. R. Nair, P. Varakantham, M. Tambe, and M. Yokoo, "Networked distributed POMDPs: A synthesis of distributed constraint optimization and POMDPs", Proc. 20th National Conference on Artificial Intelligence, 2005, pp. 133-139.

20. P. Varakantham, J. Marecki, Y. Yabu, M. Tambe, and M. Yokoo, "Letting loose a SPIDER on a network of POMDPs: Generating quality guaranteed policies", Proc. Int. Joint Conf. on Autonomous Agents and Multi-Agent Systems, 2007, pp. 817-824.

21. M. Roth, R. Simmons, and M. Veloso, "What to communicate? execution-time decision in multi-agent POMDPs", Proc. 8th Inter. Symp. on Distributed Autonomous Robotic Systems, 2006, pp. 1-10. 
22. F. Oliehoek, Matthijs Spaan, S. Whiteson, and N. Vlassis, "Exploiting locality of interaction in factored Dec-POMDPs", Proc. 7th Inter. Conf. on Autonomous Agents and Multiagent Systems, 2008, pp. 517-524.

23. P. Velagapudi, P. Varakantham, K. Sycara, and P. Scerri, "Distributed model shaping for scaling to decentralized POMDPs with hundreds of agents", Proc. Int. Joint Conf. on Autonomous Agents and Multi-Agent Systems, 2011, pp. 955-962.

24. C. Besse and B. Chaib-draa, "Parallel rollout for online solution of Dec-POMDPs", Proc. of 21st Int. FLAIRS Conference (FLAIRS-21), 2008, pp. 619-624.

25. F. Wu, S. Zilberstein, and X. Chen, "Multi-agent online planning with communication", Proc. Inter. Conf. on Automated Planning and Scheduling, 2009, pp. 321-329.

26. D.V. Pynadath and M. Tambe, "The communicative multiagent team decision problem: Analyzing teamwork theories and models", J. Artificial Intelligence Research. 16 (2002) 389-423.

27. J. Kwak, R. Yang, Z. Yin, M.E. Taylor, and M. Tambe, "Teamwork in distributed POMDPs: Execution-time coordination under model uncertainty", Proc. 10th Int. Conf. on Autonomous Agents and Multiagent Systems, 2011, pp. 1261-1262.

28. Y. Xiang, "A probabilistic framework for cooperative multi-agent distributed interpretation and optimization of communication", Artificial Intelligence. 87(1-2) (1996) 295-342.

29. R.A. Howard and J.E. Matheson, "Influence diagrams", in Readings on the Principles and Applications of Decision Analysis, eds. R.A. Howard and J.E. Matheson (Strategic Decisions Group, Menlo Park, CA, 1984) pp. 721-762.

30. L. de Silva, S. Sardina, and L. Padgham, "First principles planning in BDI systems", Proc. 8th Inter. Conf. on Autonomous Agents and Multiagent Systems, 2009, pp. 11051112 .

31. A. Kumar, B. Faltings, and A. Petcu, "Distributed constraint optimization with structured resource constraints", Proc. 8th Inter. Conf. on Autonomous Agents and Multiagent Systems, 2009, pp. 923-930. 


\section{Appendix: Notation}

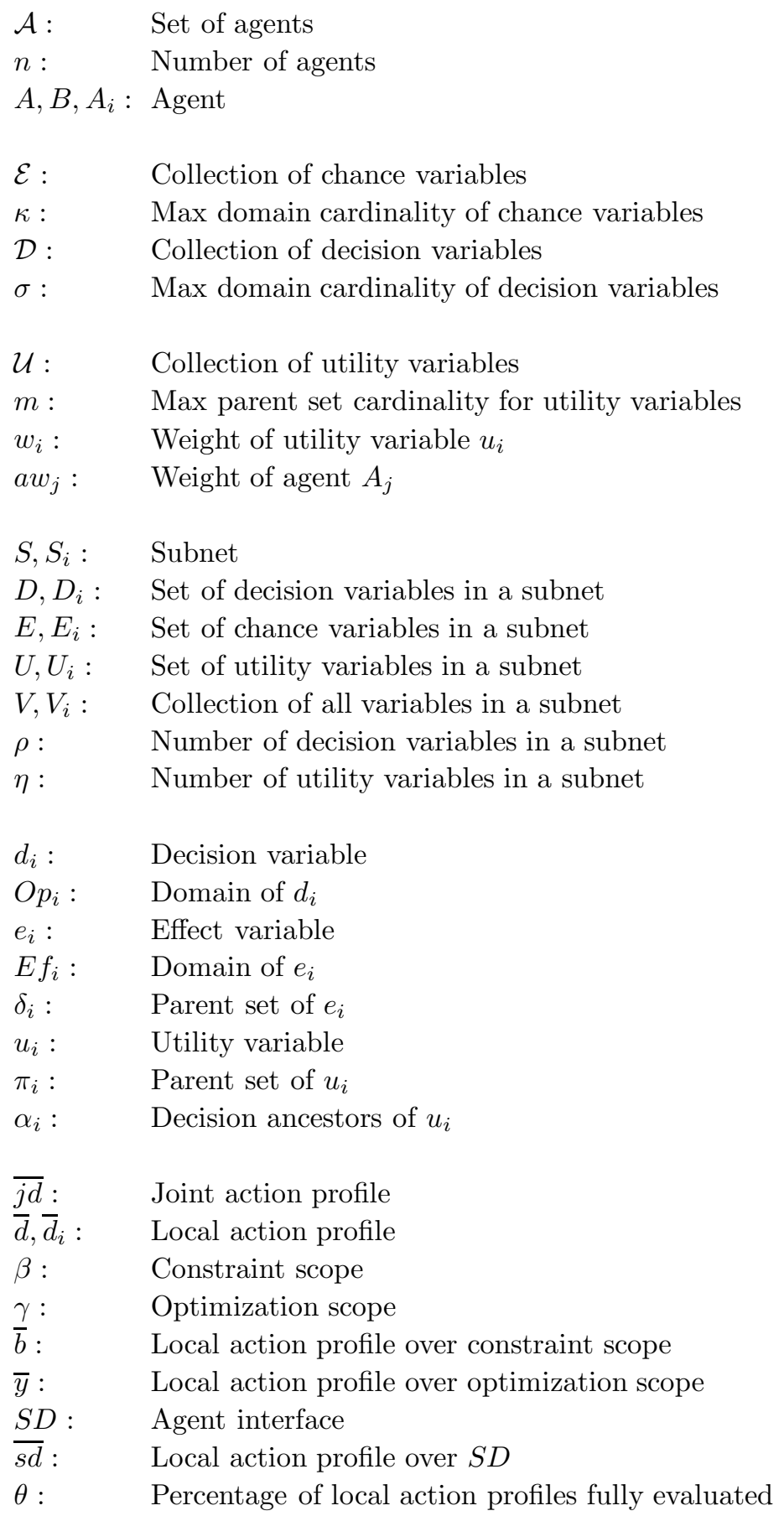

